MATHEMATICS OF COMPUTATION

Volume 72 , Number 242 , Pages $839-863$

S 0025-5718(02)01455-2

Article electronically published on June 25, 2002

\title{
A MASS FORMULA FOR UNIMODULAR LATTICES WITH NO ROOTS
}

\author{
OLIVER D. KING
}

\begin{abstract}
We derive a mass formula for $n$-dimensional unimodular lattices having any prescribed root system. We use Katsurada's formula for the Fourier coefficients of Siegel Eisenstein series to compute these masses for all root systems of even unimodular 32-dimensional lattices and odd unimodular lattices of dimension $n \leq 30$. In particular, we find the mass of even unimodular 32dimensional lattices with no roots, and the mass of odd unimodular lattices with no roots in dimension $n \leq 30$, verifying Bacher and Venkov's enumerations in dimensions 27 and 28 . We also compute better lower bounds on the number of inequivalent unimodular lattices in dimensions 26 to 30 than those afforded by the Minkowski-Siegel mass constants.
\end{abstract}

\section{INTRODUCTION}

First we review some definitions. For more information, see [9] and [21].

An $n$-dimensional lattice $\Lambda$ is the set $\mathrm{Z} v_{1}+\cdots+\mathrm{Z} v_{n}$ of all integer linear combinations of a basis $\left\{v_{1}, \ldots, v_{n}\right\}$ for $\mathrm{R}^{n}$. We associate with $\Lambda$ the Gram matrix $A$ with $i, j$-th entry the inner product $\left(v_{i}, v_{j}\right)$. (The matrix $A$ is a positive definite quadratic form, and much of what follows may be reformulated in the language of quadratic forms.) The determinant of $\Lambda$ is defined to be $\operatorname{det}(A)$, and $\Lambda$ is called unimodular if its determinant is 1 . We say that $\Lambda$ is integral if $\left(v, v^{\prime}\right)$ is an integer for all $v, v^{\prime} \in \Lambda$. Such a lattice is called even (or Type II) if $(v, v)$ is always even, and odd (or Type $I$ ) otherwise. The dual of $\Lambda$ is $\Lambda^{\prime}=\left\{v \in \mathrm{R}^{n}:(v, x) \in \mathrm{Z}\right.$ for all $\left.x \in \Lambda\right\}$, and the determinant of $\Lambda$ is the order of the finite abelian group $\Lambda^{\prime} / \Lambda$. Since we shall (with the exception of the occasional dual lattice) primarily be concerned with integral lattices, we generally omit the adjective integral in what follows. The norm of a vector $v \in \Lambda$ is defined to be $(v, v)$, the square of its length. We say that a lattice is decomposable (or reducible) if it can be written as an orthogonal direct sum of two nonzero sublattices, and is indecomposable (or irreducible) otherwise.

Each $n$-dimensional unimodular lattice $\Lambda$ has a vector $u$ such that $(u, x) \equiv(x, x)$ $(\bmod 2)$ for all $x \in \Lambda$. Such a vector is called a parity vector [9] Preface to 3rd edition, p. xxxiv], or a characteristic vector [4, or a canonical element [32. The set of parity vectors forms a coset $u+2 \Lambda$ in $\Lambda / 2 \Lambda$, and each parity vector satisfies $(u, u) \equiv n(\bmod 8)$.

Let $N$ be an $n$-dimensional lattice with Gram matrix $A$, and let $M$ be an $m$ dimensional lattice with Gram matrix $B$. We say that $N$ is represented by $M$ if

Received by the editor March 29, 2001 and, in revised form, May 8, 2001.

2000 Mathematics Subject Classification. Primary 11H55; Secondary 11E41.

This work was partially supported by grants from the NSF and the Royal Society.

(c)2002 Oliver D. King 
there exists an $m \times n$ integral matrix $X$ for which $X^{t} B X=A$. We let $r(M, N)$ denote the number of representations of $N$ by $M$. When $m=n$, we say that $M$ and $N$ are (integrally) equivalent if there exists an integral matrix $X$ with determinant \pm 1 for which $X^{t} B X=A . M$ and $N$ are in the same genus if they are equivalent over the $p$-adic integers $\mathrm{Z}_{p}$ for each prime $p$ (including $p=\infty$, for which $\mathrm{Z}_{p}=\mathrm{Q}$ ). We define $\operatorname{Aut}(N)$ to be the group of $n \times n$ integral matrices $X$ for which $X^{t} A X=A$. (Note that the definitions of $\operatorname{det}(N), r(M, N)$ and equivalence are independent of the integral bases chosen for the lattices, and so is $\operatorname{Aut}(N)$, up to isomorphism.) The theta series of a lattice $\Lambda$ is defined by

$$
\Theta_{\Lambda}(q)=\sum_{v \in \Lambda} q^{(v, v)}=\sum_{k=0}^{\infty} r(\Lambda, k) q^{k}
$$

where $q=e^{\pi i z}$.

For our purposes, a root is a vector of norm 1 or 2, and the root system of $\Lambda$ is the set of roots in $\Lambda$. The lattice generated by a root system is called a root lattice, and we define the rank of a root system to be the dimension of the corresponding root lattice. Root lattices are completely classified: they are direct sums of the irreducible root lattices Z, $A_{n}(n \geq 1), D_{n}(n \geq 4), E_{6}, E_{7}$, and $E_{8}$. We use the same notation to refer to root systems, and for brevity we sometimes write the root system

$$
n_{1} A_{1} \oplus \cdots \oplus n_{j} E_{8} \quad \text { as } A_{1}^{n_{1}} \cdots E_{8}^{n_{j}} .
$$

In this paper we are concerned with the problem of classifying unimodular lattices, and also the subproblem of classifying unimodular lattices without roots (which correspond to denser sphere-packings), up to equivalence.

The Minkowski-Siegel mass formula (see [7]) gives the sum of the reciprocals of the orders of the automorphism groups of all inequivalent lattices in a given genus. The mass constants can be used to verify that an enumeration of inequivalent lattices in a given genus is complete. They also give a lower bound for the number of inequivalent lattices in a given genus (sometimes called the class number).

In dimensions divisible by 8 there are two genera of unimodular lattices, Type I and Type II (except in dimension 0, in which there is just one lattice, of Type II). In all other dimensions there is only one genus of unimodular lattices, Type I. The lower bounds provided by the mass constants show that the number of unimodular lattices increases super-exponentially as a function of the dimension. Unimodular lattices have been completely enumerated in dimensions $n \leq 25$, but number more than 900 million in dimension 30. Unimodular lattices without roots have been completely enumerated in dimensions $n \leq 28$, but number more than $8 \times 10^{20}$ in dimension 33. In both cases, somewhere between dimension 26 and dimension 32 there is a transition from being completely classified to being numerous enough to make classification unappealing, so information about what is going on in these dimensions is of interest.

Our approach is based on a suggestion by Borcherds that the Fourier coefficients of Siegel Eisenstein series of degree $4 k$ could be used to derive something analogous to the Minkowski-Siegel mass formula, but which gives the mass of all the even unimodular lattices of dimension $8 k$ having any given root system.

We wrote a computer program that uses Katsurada's formula (see [15]) for the Fourier coefficients to calculate the masses for all possible root systems of even unimodular 32-dimensional lattices. From these masses, we used the methods of 
[9, Chapter 16] to compute the masses, for each root system, of unimodular lattices in dimensions $n \leq 30$.

In particular, we have a mass formula for those lattices which have the empty root system (that is, which have no roots). This formula verifies the known masses in dimensions $n \leq 28$, and provides new lower bounds for the number of unimodular lattices without roots in dimensions 29 to 32 .

We also used our program to compute better lower bounds on the total number of odd unimodular lattices in dimensions 26 to 30, and even unimodular lattices in dimension 32, than those gotten from the Minkowski-Siegel mass constants.

Our results may be viewed as a coarse classification of lattices, as inequivalent lattices may have the same root system. For dimensions $n \leq 23$, in which it happens to be the case that a unimodular lattice in a given genus is completely determined by its root system, our results coincide with the previously known enumerations. The same is true for the even unimodular 24-dimensional lattices (which are known as the Niemeier lattices).

\section{A MASS FORMULA FOR EVEN UNIMODULAR LATTICES HAVING ANY GIVEN ROOT SYSTEM}

Let $\Omega$ be the set of inequivalent even unimodular lattices of dimension $8 k$. We define the mass $m$ of $\Omega$ by

$$
m=\sum_{\Lambda \in \Omega} \frac{1}{|\operatorname{Aut}(\Lambda)|}
$$

By the Minkowski-Siegel mass formula, for $k>0$ we have

$$
m=\frac{\left|B_{4 k}\right|}{8 k} \prod_{j=1}^{4 k-1} \frac{\left|B_{2 j}\right|}{4 j},
$$

where $B_{i}$ is the $i$ th Bernoulli number. (See [9] and [7] for this, and for the mass formulae for other genera of lattices.)

For an $n$-dimensional lattice $N$, we define a weighted average number of representations of $N$ by the lattices $\Lambda \in \Omega$ by

$$
a(N)=\frac{1}{m} \sum_{\Lambda \in \Omega} \frac{r(\Lambda, N)}{|\operatorname{Aut}(\Lambda)|}
$$

Let $\left\{R_{1}, \ldots, R_{s}\right\}$ be the set of all the root lattices of dimension $n$ or less with no vectors of norm 1 . Each $R_{i}$ is the direct sum of lattices $A_{j}(j \geq 1), D_{j}(j \geq$ 4), $E_{6}, E_{7}$, and $E_{8}$. Let $\Omega_{i}$ be the set of lattices in $\Omega$ having root system $R_{i}$, and let $m\left(R_{i}\right)=\sum_{\Lambda \in \Omega_{i}}|\operatorname{Aut}(\Lambda)|^{-1}$ be the mass of those lattices in $\Omega$ which have root system $R_{i}$, so that $m=m\left(R_{1}\right)+\cdots+m\left(R_{s}\right)$.

Proposition 1. Let $U$ be the $s \times s$ matrix with $i, j$-th entry $r\left(R_{j}, R_{i}\right)$, let $v$ be the vector $\left(m\left(R_{1}\right), \ldots, m\left(R_{s}\right)\right)^{t}$, and let $w$ be the vector $\left(a\left(R_{1}\right), \ldots, a\left(R_{s}\right)\right)^{t}$. Then $\frac{1}{m} U v=w$. Furthermore, $U$ is invertible, so $m\left(R_{i}\right)=\left(m U^{-1} w\right)_{i}$ gives the mass of the lattices in $\Omega$ having root system $R_{i}$. 
Proof. Observe that if $R$ is any root lattice and if $S$ is the root system of a lattice $\Lambda$, then $r(\Lambda, R)=r(S, R)$, so for $j=1, \ldots, s$ we have

$$
\begin{aligned}
a\left(R_{j}\right) & =\frac{1}{m} \sum_{\Lambda \in \Omega} \frac{r\left(\Lambda, R_{j}\right)}{|\operatorname{Aut}(\Lambda)|}=\frac{1}{m} \sum_{i=1}^{s} \sum_{\Lambda \in \Omega_{i}} \frac{r\left(\Lambda, R_{j}\right)}{|\operatorname{Aut}(\Lambda)|} \\
& =\frac{1}{m} \sum_{i=1}^{s} r\left(R_{i}, R_{j}\right) \sum_{\Lambda \in \Omega_{i}} \frac{1}{|\operatorname{Aut}(\Lambda)|} \\
& =\frac{1}{m} \sum_{i=1}^{s} r\left(R_{i}, R_{j}\right) m\left(R_{i}\right) .
\end{aligned}
$$

Thus $\frac{1}{m} U v=w$. We may assume the $R_{i}$ 's are ordered so that their dimensions are non-decreasing, and so that among those with the same dimension the determinants are non-increasing. With this ordering, $r\left(R_{j}, R_{i}\right)=0$ whenever $i>j$, so $U$ is upper triangular. Since each diagonal element $r\left(R_{i}, R_{i}\right)=\left|\operatorname{Aut}\left(R_{i}\right)\right|$ is positive, we have $\operatorname{det}(U) \neq 0$, so $v=m U^{-1} w$.

Remark 2. The values $a\left(R_{j}\right)$ are the Fourier coefficients of Siegel Eisenstein series. Borcherds, Freitag and Weissauer [6] used a relation similar to $\frac{1}{m} U v=w$ to compute the coefficients of a cusp form from the known values of $m\left(R_{i}\right)$ in dimension 24 . We shall do the inverse in dimension 32: use the values of $a\left(R_{j}\right)$ to derive the values $m\left(R_{i}\right)$. We discuss how to compute $a\left(R_{j}\right)$ in Section 7 The problem of computing $r\left(R_{i}, R_{j}\right)$ is largely combinatorial; we discuss it in Section 8 .

\section{Masses of 32-Dimensional EVEn Unimodular Lattices WITH ANY GIVEN ROOT SYSTEM}

Let $m_{n}^{\mathrm{II}}(R)$ and $m_{n}^{\mathrm{I}}(R)$ denote the masses of the $n$-dimensional even and odd unimodular lattices having root system $R$, and let $m_{n}(R)$ denote their sum. Let $w(R)$ denote the order of the Weyl group of $R ; w(R)$ is the product of the orders of the Weyl groups of the irreducible components of $R$, where $w\left(A_{n}\right)=$ $(n+1) !, w\left(D_{n}\right)=2^{n-1} n !, w\left(E_{6}\right)=2^{7} 3^{4} 5 \cdot 7, w\left(E_{7}\right)=2^{10} 3^{4} 5 \cdot 7$, and $w\left(E_{8}\right)=$ $2^{14} 3^{5} 5^{2} 7$. It is sometimes more convenient to list the values of $m_{n}^{\mathrm{II}}(R) \cdot w(R)$ than it is to list the values of $m_{n}^{\mathrm{II}}(R)$ alone, but the latter can easily be recovered from the former.

We used a computer to calculate $m_{32}^{\mathrm{II}}(R)$ for each root system $R$ with $\operatorname{rank}(R) \leq$ 32 . The computation took about two weeks on a Sun Ultra 60 , running a program written in Common Lisp and compiled with Franz Inc.'s Allegro CL. (We discuss several issues related to the implementation in Sections 7 to 9)

Theorem 3. The nonzero values of $m_{32}^{I I}(R) \cdot w(R)$ with $\operatorname{rank}(R) \leq 9$ are as listed in Table 1. (The list of all 13218 nonzero values of $m_{32}^{I I}(R) \cdot w(R)$ is available electronically at [18.)

The root system $R$ of a lattice $\Lambda$ is called complete if the sublattice of $\Lambda$ generated by $R$ has finite index in $\Lambda$, or equivalently if $\operatorname{rank}(R)=\operatorname{dim}(\Lambda)$. The classification of even unimodular lattices with complete root systems is closely related to the classification of certain self-dual codes (see [33]).

Corollary 4 (Kervaire [17]). There are 119 complete root systems $R$ that occur as root systems of indecomposable 32-dimensional even unimodular lattices. 
TABlE 1. Masses of 32-dimensional even unimodular lattices

\begin{tabular}{|c|c|c|c|c|}
\hline $\operatorname{dim}$ & root system $R$ & $m_{32}^{\mathrm{II}}(R) \cdot w(R)$ & (as decimal) & comments \\
\hline 0 & $\emptyset$ & $\frac{1310037331282023326658917}{238863431761920000}$ & 5484461.50 & no roots \\
\hline 1 & $A_{1}$ & $\frac{111536168182433}{5677056}$ & 19646832.00 & \\
\hline 2 & $A_{1}^{2}$ & $\frac{72024731351193941}{1857945600}$ & 38765792.00 & \\
\hline 2 & $A_{2}$ & $\frac{1327104974887}{2939328}$ & 451499.44 & \\
\hline 3 & $A_{1}^{3}$ & $\frac{6904800898075}{124416}$ & 55497696.00 & \\
\hline 3 & $A_{1} A_{2}$ & $\frac{977951251237}{445440}$ & 2195472.50 & \\
\hline 3 & $A_{3}$ & $\frac{329127961}{74240}$ & 4433.30 & \\
\hline 4 & $A_{1}^{4}$ & $\frac{30223371257980501}{471859200}$ & 64051672.00 & \\
\hline 4 & $A_{1}^{2} A_{2}$ & $\frac{19867101805}{3456}$ & 5748582.50 & \\
\hline 4 & $A_{2}^{2}$ & $\frac{1772535692573}{42598400}$ & 41610.38 & \\
\hline 4 & $A_{1} A_{3}$ & $\frac{21073837}{768}$ & 27439.89 & \\
\hline 4 & $A_{4}$ & $\frac{8397751}{384000}$ & 21.87 & \\
\hline 4 & $D_{4}$ & $\frac{35841940559}{157212057600}$ & 0.23 & see 2 ] \\
\hline 5 & $A_{1}^{5}$ & $\frac{14457125482723}{230400}$ & 62747940.00 & \\
\hline 5 & $A_{1}^{3} A_{2}$ & $\frac{10626384230783}{995328}$ & 10676264.00 & \\
\hline 5 & $A_{1} A_{2}^{2}$ & $\frac{673556587}{2560}$ & 263108.06 & \\
\hline 5 & $A_{1}^{2} A_{3}$ & $\frac{200386803709}{2211840}$ & 90597.34 & \\
\hline 5 & $A_{2} A_{3}$ & $\frac{8085187}{5760}$ & 1403.68 & \\
\hline 5 & $A_{1} A_{4}$ & $\frac{46917823}{269568}$ & 174.05 & \\
\hline 5 & $A_{1} D_{4}$ & $\frac{473}{240}$ & 1.97 & \\
\hline 5 & $A_{5}$ & $\frac{73}{960}$ & 0.08 & \\
\hline 5 & $D_{5}$ & $\frac{433}{3317760}$ & 0.00 & see 2 ] \\
\hline 6 & $A_{1}^{6}$ & $\frac{355695555290333}{6635520}$ & 53604776.00 & \\
\hline 6 & $A_{1}^{4} A_{2}$ & $\frac{22484458507}{1440}$ & 15614208.00 & \\
\hline 6 & $A_{1}^{2} A_{2}^{2}$ & $\frac{16820220686833}{19169280}$ & 877457.06 & \\
\hline 6 & $A_{1}^{3} A_{3}$ & $\frac{5452147363}{25920}$ & 210345.19 & \\
\hline 6 & $A_{2}^{3}$ & $\frac{9785018477}{1866240}$ & 5243.17 & \\
\hline 6 & $A_{1} A_{2} A_{3}$ & $\frac{680633479}{61440}$ & 11078.02 & \\
\hline 6 & $A_{1}^{2} A_{4}$ & $\frac{11264777}{15360}$ & 733.38 & \\
\hline 6 & $A_{3}^{2}$ & $\frac{323400013}{20447232}$ & 15.82 & \\
\hline 6 & $A_{1}^{2} D_{4}$ & $\frac{622763}{69120}$ & 9.01 & \\
\hline 6 & $A_{2} A_{4}$ & $\frac{6059}{512}$ & 11.83 & \\
\hline 6 & $A_{2} D_{4}$ & $\frac{344077}{2446080}$ & 0.14 & \\
\hline 6 & $A_{1} A_{5}$ & $\frac{123853}{159744}$ & 0.78 & \\
\hline 6 & $A_{1} D_{5}$ & $\frac{1}{648}$ & 0.00 & \\
\hline 6 & $A_{6}$ & $\frac{1}{4608}$ & 0.00 & \\
\hline
\end{tabular}


TABLE 1. Masses of 32-dimensional even unimodular lattices (continued)

\begin{tabular}{|c|c|c|c|c|}
\hline $\operatorname{dim}$ & root system $R$ & $m_{32}^{\mathrm{II}}(R) \cdot w(R)$ & (as decimal) & comments \\
\hline 6 & $D_{6}$ & $\frac{1}{18720000}$ & 0.00 & unique \\
\hline 6 & $E_{6}$ & $\frac{1}{1268047872}$ & 0.00 & unique \\
\hline 7 & $A_{1}^{7}$ & $\frac{23307759701}{576}$ & 40464860.00 & \\
\hline 7 & $A_{1}^{5} A_{2}$ & $\frac{290429642677}{15360}$ & 18908180.00 & \\
\hline 7 & $A_{1}^{3} A_{2}^{2}$ & $\frac{32712111919}{16128}$ & 2028280.70 & \\
\hline 7 & $A_{1}^{4} A_{3}$ & $\frac{491983248817}{1290240}$ & 381311.40 & \\
\hline 7 & $A_{1} A_{2}^{3}$ & $\frac{641243179}{15360}$ & 41747.60 & \\
\hline 7 & $A_{1}^{2} A_{2} A_{3}$ & $\frac{528426689}{11520}$ & 45870.37 & \\
\hline 7 & $A_{1}^{3} A_{4}$ & $\frac{828763}{384}$ & 2158.24 & \\
\hline 7 & $A_{2}^{2} A_{3}$ & $\frac{238819303}{552960}$ & 431.89 & \\
\hline 7 & $A_{1}^{3} D_{4}$ & $\frac{2242333}{77760}$ & 28.84 & \\
\hline 7 & $A_{1} A_{3}^{2}$ & $\frac{2750969}{17920}$ & 153.51 & \\
\hline 7 & $A_{1} A_{2} A_{4}$ & $\frac{57178267}{483840}$ & 118.18 & \\
\hline 7 & $A_{1}^{2} A_{5}$ & $\frac{24071}{5760}$ & 4.18 & \\
\hline 7 & $A_{1} A_{2} D_{4}$ & $\frac{959}{640}$ & 1.50 & \\
\hline 7 & $A_{3} A_{4}$ & $\frac{297043}{860160}$ & 0.35 & \\
\hline 7 & $A_{2} A_{5}$ & $\frac{51383}{774144}$ & 0.07 & \\
\hline 7 & $A_{3} D_{4}$ & $\frac{133}{30720}$ & 0.00 & \\
\hline 7 & $A_{1}^{2} D_{5}$ & $\frac{11}{1152}$ & 0.01 & \\
\hline 7 & $A_{1} A_{6}$ & $\frac{521}{188160}$ & 0.00 & \\
\hline 7 & $A_{2} D_{5}$ & $\frac{1}{6912}$ & 0.00 & \\
\hline 7 & $A_{7}$ & $\frac{1}{1376256}$ & 0.00 & \\
\hline 8 & $A_{1}^{8}$ & $\frac{2005621383142854931}{73987522560}$ & 27107562.00 & \\
\hline 8 & $A_{1}^{6} A_{2}$ & $\frac{2015000372681}{103680}$ & 19434804.00 & \\
\hline 8 & $A_{1}^{4} A_{2}^{2}$ & $\frac{1327413084613}{368640}$ & 3600838.50 & \\
\hline 8 & $A_{1}^{5} A_{3}$ & $\frac{980176289}{1728}$ & 567231.70 & \\
\hline 8 & $A_{1}^{2} A_{2}^{3}$ & $\frac{13761649541}{80640}$ & 170655.37 & \\
\hline 8 & $A_{1}^{3} A_{2} A_{3}$ & $\frac{42164571593}{322560}$ & 130718.54 & \\
\hline 8 & $A_{2}^{4}$ & $\frac{176324027322323}{180592312320}$ & 976.37 & \\
\hline 8 & $A_{1}^{4} A_{4}$ & $\frac{9542951321}{1935360}$ & 4930.84 & \\
\hline 8 & $A_{1} A_{2}^{2} A_{3}$ & $\frac{691208023}{161280}$ & 4285.76 & \\
\hline 8 & $A_{1}^{4} D_{4}$ & $\frac{371231029}{5160960}$ & 71.93 & \\
\hline 8 & $A_{1}^{2} A_{3}^{2}$ & $\frac{6071138573}{7741440}$ & 784.24 & \\
\hline 8 & $A_{1}^{2} A_{2} A_{4}$ & $\frac{1655917}{2688}$ & 616.04 & \\
\hline 8 & $A_{2} A_{3}^{2}$ & $\frac{1794539}{120960}$ & 14.84 & \\
\hline 8 & $A_{1}^{3} A_{5}$ & $\frac{2245489}{143360}$ & 15.66 & \\
\hline
\end{tabular}


TABLE 1. Masses of 32-dimensional even unimodular lattices (continued)

\begin{tabular}{|c|c|c|c|c|}
\hline $\operatorname{dim}$ & root system $R$ & $m_{32}^{\mathrm{II}}(R) \cdot w(R)$ & (as decimal) & comments \\
\hline 8 & $A_{1}^{2} A_{2} D_{4}$ & $\frac{6113}{720}$ & 8.49 & \\
\hline 8 & $A_{2}^{2} A_{4}$ & $\frac{237}{40}$ & 5.93 & \\
\hline 8 & $A_{1} A_{3} A_{4}$ & $\frac{200995}{48384}$ & 4.15 & \\
\hline 8 & $A_{2}^{2} D_{4}$ & $\frac{18917}{266112}$ & 0.07 & \\
\hline 8 & $A_{1} A_{2} A_{5}$ & $\frac{7739}{8960}$ & 0.86 & \\
\hline 8 & $A_{1}^{3} D_{5}$ & $\frac{829}{20160}$ & 0.04 & \\
\hline 8 & $A_{1} A_{3} D_{4}$ & $\frac{353}{6720}$ & 0.05 & \\
\hline 8 & $A_{1}^{2} A_{6}$ & $\frac{2287}{120960}$ & 0.02 & \\
\hline 8 & $A_{4}^{2}$ & $\frac{432673039}{170311680000}$ & 0.00 & \\
\hline 8 & $A_{3} A_{5}$ & $\frac{1049}{483840}$ & 0.00 & \\
\hline 8 & $A_{1} A_{2} D_{5}$ & $\frac{43}{20160}$ & 0.00 & \\
\hline 8 & $A_{2} A_{6}$ & $\frac{1}{3780}$ & 0.00 & \\
\hline 8 & $A_{4} D_{4}$ & $\frac{1}{15360}$ & 0.00 & \\
\hline 8 & $D_{4}^{2}$ & $\frac{1867}{1937768448}$ & 0.00 & \\
\hline 8 & $A_{3} D_{5}$ & $\frac{19}{1935360}$ & 0.00 & \\
\hline 8 & $A_{1}^{2} D_{6}$ & $\frac{607}{85155840}$ & 0.00 & \\
\hline 8 & $A_{1} A_{7}$ & $\frac{5}{1064448}$ & 0.00 & \\
\hline 8 & $A_{8}$ & $\frac{1}{185794560}$ & 0.00 & \\
\hline 8 & $A_{2} E_{6}$ & $\frac{1}{277136640}$ & 0.00 & unique \\
\hline 8 & $D_{8}$ & $\frac{1}{1002795171840}$ & 0.00 & odd Leech \\
\hline 8 & $E_{8}$ & $\frac{1}{8315553613086720000}$ & 0.00 & Leech \\
\hline 9 & $A_{1}^{9}$ & $\frac{4668288705497}{290304}$ & 16080690.00 & \\
\hline 9 & $A_{1}^{7} A_{2}$ & $\frac{5526347655971}{322560}$ & 17132774.00 & \\
\hline 9 & $A_{1}^{5} A_{2}^{2}$ & $\frac{740728763}{144}$ & 5143950.00 & \\
\hline 9 & $A_{1}^{6} A_{3}$ & $\frac{1568559349553}{2211840}$ & 709164.94 & \\
\hline 9 & $A_{1}^{3} A_{2}^{3}$ & $\frac{1368567316381}{2903040}$ & 471425.60 & \\
\hline 9 & $A_{1}^{4} A_{2} A_{3}$ & $\frac{1271443287}{4480}$ & 283804.30 & \\
\hline 9 & $A_{1} A_{2}^{4}$ & $\frac{23738023}{2560}$ & 9272.67 & \\
\hline 9 & $A_{1}^{5} A_{4}$ & $\frac{2142275851}{233280}$ & 9183.28 & \\
\hline 9 & $A_{1}^{2} A_{2}^{2} A_{3}$ & $\frac{927969995}{43008}$ & 21576.68 & \\
\hline 9 & $A_{1}^{5} D_{4}$ & $\frac{158461}{1080}$ & 146.72 & \\
\hline 9 & $A_{1}^{3} A_{3}^{2}$ & $\frac{36859871}{13440}$ & 2742.55 & \\
\hline 9 & $A_{1}^{3} A_{2} A_{4}$ & $\frac{237492443}{107520}$ & 2208.82 & \\
\hline 9 & $A_{2}^{3} A_{3}$ & $\frac{3078967}{17280}$ & 178.18 & \\
\hline 9 & $A_{1}^{4} A_{5}$ & $\frac{457601}{10080}$ & 45.40 & \\
\hline 9 & $A_{1}^{3} A_{2} D_{4}$ & $\frac{21454849}{645120}$ & 33.26 & \\
\hline
\end{tabular}


TABLE 1. Masses of 32-dimensional even unimodular lattices (continued)

\begin{tabular}{|c|c|c|c|c|}
\hline $\operatorname{dim}$ & root system $R$ & $m_{32}^{\mathrm{II}}(R) \cdot w(R)$ & (as decimal) & comments \\
\hline 9 & $A_{1} A_{2} A_{3}^{2}$ & $\frac{6031423}{32256}$ & 186.99 & \\
\hline 9 & $A_{1} A_{2}^{2} A_{4}$ & $\frac{989231}{13440}$ & 73.60 & \\
\hline 9 & $A_{1}^{2} A_{3} A_{4}$ & $\frac{1223851}{46080}$ & 26.56 & \\
\hline 9 & $A_{1}^{2} A_{2} A_{5}$ & $\frac{6696269}{1182720}$ & 5.66 & \\
\hline 9 & $A_{1} A_{2}^{2} D_{4}$ & $\frac{2927}{2880}$ & 1.02 & \\
\hline 9 & $A_{3}^{3}$ & $\frac{31211779}{163296000}$ & 0.19 & \\
\hline 9 & $A_{1}^{4} D_{5}$ & $\frac{1029287}{7464960}$ & 0.14 & \\
\hline 9 & $A_{1}^{2} A_{3} D_{4}$ & $\frac{66941}{184320}$ & 0.36 & \\
\hline 9 & $A_{2} A_{3} A_{4}$ & $\frac{3827}{8064}$ & 0.47 & \\
\hline 9 & $A_{1}^{3} A_{6}$ & $\frac{34339}{384000}$ & 0.09 & \\
\hline 9 & $A_{2}^{2} A_{5}$ & $\frac{783383}{13063680}$ & 0.06 & \\
\hline 9 & $A_{1} A_{4}^{2}$ & $\frac{1021}{32256}$ & 0.03 & \\
\hline 9 & $A_{2} A_{3} D_{4}$ & $\frac{1}{252}$ & 0.00 & \\
\hline 9 & $A_{1}^{2} A_{2} D_{5}$ & $\frac{37}{2520}$ & 0.01 & \\
\hline 9 & $A_{1} A_{3} A_{5}$ & $\frac{4813}{129024}$ & 0.04 & \\
\hline 9 & $A_{1} A_{2} A_{6}$ & $\frac{547}{126720}$ & 0.00 & \\
\hline 9 & $A_{1} A_{4} D_{4}$ & $\frac{1}{1260}$ & 0.00 & \\
\hline 9 & $A_{2}^{2} D_{5}$ & $\frac{1}{8000}$ & 0.00 & \\
\hline 9 & $A_{1}^{2} A_{7}$ & $\frac{43}{430080}$ & 0.00 & \\
\hline 9 & $A_{1} A_{3} D_{5}$ & $\frac{1}{15840}$ & 0.00 & \\
\hline 9 & $A_{4} A_{5}$ & $\frac{13}{295680}$ & 0.00 & \\
\hline 9 & $A_{3} A_{6}$ & $\frac{1}{466560}$ & 0.00 & \\
\hline 9 & $A_{5} D_{4}$ & $\frac{1}{1512000}$ & 0.00 & \\
\hline 9 & $A_{2} A_{7}$ & $\frac{1}{504000}$ & 0.00 & \\
\hline 9 & $A_{1}^{3} E_{6}$ & $\frac{1}{1512000}$ & 0.00 & unique \\
\hline 9 & $A_{1} A_{2} D_{6}$ & $\frac{1}{645120}$ & 0.00 & \\
\hline 9 & $A_{4} D_{5}$ & $\frac{1}{1774080}$ & 0.00 & \\
\hline 9 & $A_{1} A_{8}$ & $\frac{1}{20401920}$ & 0.00 & \\
\hline 9 & $D_{4} D_{5}$ & $\frac{1}{41287680}$ & 0.00 & \\
\hline 9 & $A_{3} D_{6}$ & $\frac{1}{52254720}$ & 0.00 & \\
\hline 9 & $A_{1}^{2} D_{7}$ & $\frac{1}{177408000}$ & 0.00 & unique \\
\hline 9 & $A_{3} E_{6}$ & $\frac{1}{489646080}$ & 0.00 & unique \\
\hline 9 & $A_{9}$ & $\frac{1}{3592512000}$ & 0.00 & \\
\hline 9 & $A_{2} E_{7}$ & $\frac{1}{991533312000}$ & 0.00 & unique \\
\hline 9 & $D_{9}$ & $\frac{1}{84610842624000}$ & 0.00 & shorter Leech \\
\hline
\end{tabular}


Proof. There are 143 root systems $R$ for which $\operatorname{rank}(R)=32$ and $m_{32}^{\mathrm{II}}(R)>0$. Of these, the root system $D_{16}^{2}$ and the 23 root systems containing $E_{8}$ correspond to decomposable lattices. The remaining 119 root systems correspond to indecomposable lattices.

Kervaire 17, extending the work of Koch and Venkov 24, has proven a stronger result: there are exactly 132 indecomposable even unimodular 32-dimensional lattices with complete root systems, with 119 different root systems occurring.

Corollary 5. There are at least ten million 32-dimensional even unimodular lattices without roots.

Proof. Each such lattice has at least two automorphisms, so the number of such lattices is at least $2 \times m_{32}^{\mathrm{II}}(\emptyset)=1.096 \times 10^{7}$. We will have more to say about lattices without roots is Section 5 .

Remark 6. If there is a single even unimodular 32-dimensional lattice with root system $R$, then the corresponding value $m_{32}^{\mathrm{II}}(R) \cdot w(R)$ is of the form $1 / q$ for some $q \in \mathrm{N}$; the converse often holds (but not always: for $R=A_{1}^{7} A_{2}^{3} A_{3} D_{7}, m_{32}^{\mathrm{II}}(R) \cdot w(R)$ splits as $1 / 4=1 / 12+1 / 6$; see [4]). We give one example below, and shall see more examples in Section 4 (Niemeier lattices) and Section 5 (unimodular lattices with no roots). The last column of Table 1 labels as "unique" those lattices whose uniqueness follows from [4, and in some cases gives the name of the lower dimensional lattice that when glued to $R$ produces the unique even unimodular 32-dimensional lattice with root system $R$.

Example 7. In 12], Elkies and Gross construct a 26-dimensional even lattice $L_{0}$ of determinant 3 with no roots, for which $\left|\operatorname{Aut}\left(L_{0}\right)\right|=2^{13} 3^{5} 7^{2} 13=1268047872$, and sketch a proof of its uniqueness that uses Euclidean lattices. (Borcherds [4] Chapter 5.7] had previously proved its existence and uniqueness using Lorentzian lattices.) By 4, even 26-dimensional lattices of determinant 3 with no roots are in one-to-one correspondence with even unimodular 32-dimensional lattices having root system $E_{6}$, where the order of the automorphism group of the 32-dimensional lattice is $w\left(E_{6}\right)$ times the order the automorphism group of the corresponding 26dimensional lattice. Since $m_{32}^{\mathrm{II}}\left(E_{6}\right) \cdot w\left(E_{6}\right)=\left|\operatorname{Aut}\left(L_{0}\right)\right|^{-1}, L_{0}$ must be the unique even 26-dimensional lattice of determinant 3 with no roots, and there must also be a unique 32-dimensional even unimodular lattice with root system $E_{6}$. (This also follows from the classification of 27-dimensional unimodular lattices with no roots in [2], as the one-to-one correspondence mentioned above also extends to 27dimensional unimodular lattices with no roots and with a parity vector of norm 3 [4].)

Remark 8. In an earlier draft we pointed out the large mass of lattices with root systems $A_{1}^{k}$ for small $k$, and remarked that roots seem to have a propensity for being orthogonal to one another. Peters has since sent us a preprint [31] explaining this: he observes that the sum of the masses $m_{32}^{\mathrm{II}}(\emptyset)+\sum_{k=1}^{32} m_{32}^{\mathrm{II}}\left(A_{1}^{k}\right)$ in [18] is $97.25 \%$ of the total mass of the genus of even unimodular 32-dimensional lattices, and shows that a lower bound of $97.11 \%$ of the total mass can be derived from the small size of the Fourier coefficient corresponding to $A_{2}$ in the Siegel Eisenstein series of degree 2 and weight 16 . The idea is that an even unimodular lattice represents $A_{2}$ if and only if it has non-orthogonal roots $u$ and $v$ (with $u \neq \pm v$ ). 


\section{Masses of Unimodular lattices of Dimension $n \leq 30$ WITH ANY GIVEN ROOT SYSTEM}

In [9, Chapter 16], Conway and Sloane describe a correspondence between unimodular lattices of dimension $n \leq 23$ and orbits of norm 4 vectors in even unimodular 24-dimensional lattices. They use this correspondence to produce, from the list of Niemeier lattices, a list of the root systems and automorphism group orders of all unimodular lattices of dimension $n \leq 23$. Unimodular lattices of dimension $n \leq 31$ likewise correspond to orbits of norm 4 vectors in even unimodular 32-dimensional lattices, and we can use this correspondence to compute $m_{n}(R)$ for all root systems $R$ and all $n \leq 30$.

Let $v=2 e$ be a vector of norm 4 in an even unimodular lattice $\Lambda$ of dimension $n=32$. Then $L_{31}=\left\{x \in \Lambda^{\perp}: x+n e \in \Lambda\right.$ for some $\left.n \in \mathrm{Z}\right\}$ is an odd 31dimensional unimodular lattice, and $\left|\operatorname{Aut}\left(L_{31}\right)\right|=2|\operatorname{Aut}(\Lambda)| / c(v)$, where $c(v)$ is the number of images of $v$ under $\operatorname{Aut}(\Lambda)$. If the lattice $L_{31}$ has exactly $2 k$ vectors of norm 1, we can write $L_{31}=L_{31-k} \oplus \mathrm{Z}^{k}$, where $L_{31-k}$ has minimal norm 2 and $\left|\operatorname{Aut}\left(L_{31-k}\right)\right|=\left|\operatorname{Aut}\left(L_{31}\right)\right| /\left(2^{k} k !\right)$. (We shall call $L_{31-k}$ the reduced lattice corresponding to $\Lambda$ and $v$.) This construction gives a one-to-one correspondence between orbits of norm 4 vectors in 32-dimensional even unimodular lattices and unimodular lattices with no vectors of norm 1 in dimensions less than 32 . (See [9] or [4]).

We shall mainly be concerned with norm 4 vectors $v$ that are the sum of two orthogonal roots $r$ and $s$. For such vectors, knowing just the root system of $\Lambda$ allows us to compute the dimension and the root system of the reduced lattice $L_{31-k}$. Some useful information, distilled from [9, Table 16.8], is provided in the table below.

\begin{tabular}{lllclll}
$R$ & $\# r$ & $\hat{R}$ & shape of $v$ & $\# v$ & $\tilde{R}$ & $\operatorname{dim}\left(L_{31-k}\right)$ \\
\hline$A_{n}$ & $4\left(\begin{array}{c}n+1 \\
2\end{array}\right)$ & $A_{n-2}$ & any & $6\left(\begin{array}{c}n+1 \\
4\end{array}\right)$ & $A_{n-4}$ & 29 \\
$D_{4}$ & 24 & $A_{1}^{3}$ & any & 24 & $\emptyset$ & 28 \\
$D_{n}(n \geq 5)$ & $4\left(\begin{array}{c}n \\
2\end{array}\right)$ & $D_{n-2}$ & $\left( \pm 1^{4}, 0^{n-4}\right)$ & $16\left(\begin{array}{c}n \\
4\end{array}\right)$ & $D_{n-4}$ & 28 \\
$E_{6}$ & 72 & $A_{5}$ & any & 270 & $\emptyset$ & 27 \\
$E_{7}$ & 126 & $D_{6}$ & any & 756 & $A_{1}$ & 26 \\
$E_{8}$ & 240 & $E_{7}$ & any & 2160 & $\emptyset$ & 24
\end{tabular}

The interpretation of this table is as follows:

Case 1: $r$ and $s$ are from different components $R$ and $S$ of the root system of $\Lambda$. Then the reduced lattice $L_{31-k}$ has dimension 30, and has the same root system as $\Lambda$, except for the components $R$ and $S$ from which $r$ and $s$ are taken, which are transformed to $\hat{R}$ and $\hat{S}$ as given in the third column. The second column gives the number of roots $\# r$ in each component $R$, from which we can compute the number of vectors $v=r+s$ with $r \in R$ and $s \in S$.

Case 2: $r$ and $s$ are from the same component $R$ of the root system of $\Lambda$. Then the dimension of the reduced lattice is given in the last column, and the root system of $L_{32-k}$ is the same as that of $\Lambda$ except the component $R$ is replaced by $\tilde{R}$. Note that for $R=D_{n}$ with $n \geq 5, \tilde{R}$ and $\operatorname{dim}\left(L_{32-k}\right)$ depend on the shape of $v$. The column headed \#v gives the number of norm 4 vectors of that shape in the component $R$.

Remark 9. Norm 4 vectors that are not the sum of two orthogonal roots correspond to reduced lattices of dimension 31. For this reason we have not computed the 
mass of unimodular 31-dimensional lattices with root system $R$. (That mass could be computed from the mass of even unimodular 40-dimensional lattices with root system $R \oplus D_{9}$.)

Computing the number $c(v)$ (and hence the order of $\operatorname{Aut}\left(L_{31-k}\right)$ in terms of the order of $\operatorname{Aut}(\Lambda))$ sometimes requires additional information about $\Lambda$, such as whether there are automorphisms of $\Lambda$ that permute the components of its root system with multiplicity greater than one. But for our purposes this is not an impediment, as we demonstrate in the following example.

Example 10. Let $\Lambda$ be an even unimodular 32-dimensional lattice with root system $A_{1}^{4} D_{5}$ and let $v=s+t$, where $s$ is a root from one of the four components $A_{1}$ and $t$ is a root from the component $D_{5}$. Then the corresponding reduced lattice $L_{31-k}$ is 30-dimensional, has root system $A_{1}^{4} A_{3}$ (since $D_{3}=A_{3}$ ), and has automorphism group order $\left|\operatorname{Aut}\left(L_{30}\right)\right|=(2|\operatorname{Aut}(\Lambda)| / c(v)) /\left(2^{1} \cdot 1 !\right)=|\operatorname{Aut}(\Lambda)| / c(v)$. Since each component $A_{1}$ has 2 roots and the component $D_{5}$ has 40 roots, there are $4 \cdot 2 \cdot 40=320$ such vectors $v$, which form anywhere from one to four orbits under Aut $(\Lambda)$, depending on whether there are any automorphisms permuting the components $A_{1}$. Because of this ambiguity, we do not know exactly what $c(v)$ is. But suppose the 320 vectors break into $m$ orbits, $V_{1}, \ldots, V_{m}$, with representatives $v_{1}, \ldots, v_{m}$. Then

$$
\sum_{i=1}^{m} c\left(v_{i}\right)=\sum_{i=1}^{m}\left|V_{i}\right|=320
$$

so the total mass of the lattices that correspond to the lattice $\Lambda$ and any of these vectors $v$ is

$$
\sum_{i=1}^{m} \frac{c\left(v_{i}\right)}{|\operatorname{Aut}(\Lambda)|}=\frac{320}{|\operatorname{Aut}(\Lambda)|} .
$$

Since from Table 1 the mass of all 32-dimensional lattices $\Lambda_{j}$ with root system $A_{1}^{4} D_{5}$ is $\sum_{j}\left|\operatorname{Aut}\left(\Lambda_{j}\right)\right|^{-1}=1029287 / 7464960$, these lattices contribute $\sum_{j} 320\left|\operatorname{Aut}\left(\Lambda_{j}\right)\right|^{-1}$ $=320 \cdot 1029287 / 7464960$ towards the total mass of 30-dimensional unimodular lattices with root system $A_{1}^{4} A_{3}$. By similarly accounting for contributions from orbits of norm 4 vectors in even unimodular 32-dimensional lattices with other root systems, we can compute the exact mass of the 30-dimensional unimodular lattices with root system $A_{1}^{4} A_{3}$.

We have, in this manner, computed the mass of $n$-dimensional unimodular lattices having any given root system for all $n \leq 30$. We have not provided a table of these masses (as they can easily be derived from the table of masses of 32dimensional even unimodular lattices with any given root system in [18]), but we will use these masses in Section [6 to find lower bounds on the class numbers of unimodular lattices in dimensions up to 30 .

Example 11. Even unimodular 24-dimensional lattices with root system $R$ correspond to even unimodular 32-dimensional lattices with root system $R \oplus E_{8}$, and $m_{24}^{\mathrm{II}}(R)=m_{32}^{\mathrm{II}}\left(R \oplus E_{8}\right) \cdot w\left(E_{8}\right) \cdot c$, where $c$ is the multiplicity of $E_{8}$ in $R \oplus E_{8}$. We see from the table in [18] that there are 24 root systems of the form $R \oplus E_{8}$ for which $m_{32}^{\mathrm{II}}\left(R \oplus E_{8}\right)>0$. Since even unimodular 24-dimensional lattices happen to be uniquely determined by their root systems, the corresponding values of $m_{24}^{\mathrm{II}}(R)$ 
are precisely of the form $|\operatorname{Aut}(\Lambda)|^{-1}$, where $\Lambda$ is the Niemeier lattice having root system $R$.

\section{MASS FORMula FOR Unimodular lattices With NO ROOTS}

Recall that $m_{n}(\emptyset)$ denotes the mass of the $n$-dimensional unimodular lattices with no roots. By considering all the orbits of norm 4 vectors $v=r+s$ in even unimodular 32-dimensional lattices that correspond to reduced lattices with no roots, we have

$$
\begin{aligned}
m_{n}(\emptyset) & =m_{32}^{\mathrm{II}}\left(D_{32-n}\right) w\left(D_{32-n}\right) \quad \text { for } n \leq 26, n \neq 24, \\
m_{24}(\emptyset) & =m_{32}^{\mathrm{II}}\left(D_{8}\right) w\left(D_{8}\right)+m_{32}^{\mathrm{II}}\left(E_{8}\right) w\left(E_{8}\right), \\
m_{27}(\emptyset) & =m_{32}^{\mathrm{II}}\left(D_{5}\right) w\left(D_{5}\right)+m_{32}^{\mathrm{II}}\left(E_{6}\right) w\left(E_{6}\right), \\
m_{28}(\emptyset) & =m_{32}^{\mathrm{II}}\left(D_{4}\right) 3 w\left(D_{4}\right)+m_{32}^{\mathrm{II}}\left(D_{5}\right) w\left(D_{5}\right), \\
m_{29}(\emptyset) & =m_{32}^{\mathrm{II}}\left(A_{3}\right) w\left(A_{3}\right)+m_{32}^{\mathrm{II}}\left(A_{4}\right) w\left(A_{4}\right), \\
m_{30}(\emptyset) & =m_{32}^{\mathrm{II}}\left(A_{1}^{2}\right) w\left(A_{1}^{2}\right)+m_{32}^{\mathrm{II}}\left(A_{1} A_{2}\right) w\left(A_{1} A_{2}\right)+m_{32}^{\mathrm{II}}\left(A_{2}^{2}\right) w\left(A_{2}^{2}\right) .
\end{aligned}
$$

These masses $m_{n}(\emptyset)$ may be computed by looking up the values of $m_{32}^{\mathrm{II}}(R)$ in Table 1 and [18; the results are listed in Table 2, split into $m_{n}^{\mathrm{I}}(\emptyset)$ and $m_{n}^{\mathrm{II}}(\emptyset)$ for $n$ divisible by 8 . Table 2 also includes lower bounds on $m_{n}^{\mathrm{I}}(\emptyset)$ for $n=31$ and 32 , which we explain below.

Suppose $\Lambda$ is an even unimodular 32-dimensional lattice with no roots. It follows from a theta function argument as in [9, Theorem 7.17] that $\Lambda$ has 146880 vectors $v$ of norm 4 . For each orbit of these vectors, the corresponding reduced lattice is 31-dimensional and has no roots. Thus these lattices $\Lambda$ contribute

$$
\frac{146880}{2} m_{32}^{\mathrm{II}}(\emptyset)=\frac{22270634631794396553201589}{55292461056000} \approx 4.03 \times 10^{11}
$$

towards the mass of 31-dimensional lattices with no roots. There are almost certainly additional contributions from norm 4 glue vectors $v$ in even unimodular 32-dimensional lattices with root systems $A_{1}^{k}$ for some $k$, but we will not attempt to account for these. We can restate this in terms of parity vectors:

Proposition 12. The mass of 31-dimensional unimodular lattices with no roots and with no parity vectors of norm 7 is $(146880 / 2) m_{32}^{I I}(\emptyset)$.

Proof. Let $L$ be a 31-dimensional unimodular lattice with no roots, and $\Lambda$ the corresponding 32-dimensional even unimodular lattice. As in [9, p. 414], $\Lambda$ is equal to $\left\{x+y \mid x \in L^{i}, y \in \frac{i}{2}+2 \mathrm{Z}\right.$, for $\left.i=0,1,2,3\right\} \subset\left(L^{0}\right)^{\prime} \oplus \frac{1}{2} \mathrm{Z}$, where $L^{0}$ is the sublattice of $L$ consisting of vectors of even norm; $L^{0}, L^{1}, L^{2}$ and $L^{3}$ are the cosets of $L^{0}$ in its dual $\left(L^{0}\right)^{\prime}$; and $L^{0} \cup L^{2}=L$. The only ways the vector $x+y$ in $\Lambda$ can have norm 2 is if $y=0$ and $(x, x)=2$ with $x \in L^{0}$, if $y=1$ and $(x, x)=1$ with $x \in L^{2}$, or if $y= \pm 1 / 2$ and $(x, x)=7 / 4$ with $x \in L^{1}$ or $x \in L^{3}$. In the first two cases $x$ would be in $L$, which would contradict $L$ having no roots. Thus $\Lambda$ has a root if and only if there is a vector $x$ of norm $7 / 4$ in $L^{1} \cup L^{3}$. But in this case $x$ is in $\left(L^{0}\right)^{\prime} \backslash L$ - the so-called shadow of $L$ - and $2 x$ has norm 7 . Since the parity vectors of $L$ are precisely twice the shadow vectors [9] Preface to 3rd edition, p. xxxiv], $L$ has a parity vector of norm 7 if and only if $\Lambda$ has a root. (The 31-dimensional unimodular lattices with no roots and no parity vectors of norm 7 must have parity vectors of norm 15 by [11].) 
TABle 2. Masses of $n$-dimensional unimodular lattices without roots. Odd lattices appear above, even lattices below.

\begin{tabular}{|c|c|c|c|}
\hline$n$ & $m_{n}^{\mathrm{I}}(\emptyset)$ & (as decimal) & number of lattices \\
\hline $0-22$ & 0 & 0 & 0 \\
\hline 23 & $\frac{1}{84610842624000}$ & $1.18 \times 10^{-14}$ & (shorter Leech lattice) \\
\hline 24 & $\frac{1}{1002795171840}$ & $9.97 \times 10^{-13}$ & (odd Leech lattice) \\
\hline 25 & 0 & 0 & 0 \\
\hline 26 & $\frac{1}{18720000}$ & $5.34 \times 10^{-8}$ & (classified in [4]) \\
\hline 27 & $\frac{206867}{1585059840}$ & $1.31 \times 10^{-4}$ & 3 (classified in [2]) \\
\hline 28 & $\frac{17924389897}{26202009600}$ & $6.84 \times 10^{-1}$ & 38 (classified in [2]) \\
\hline 29 & $\frac{49612728929}{11136000}$ & $4.46 \times 10^{3}$ & more than 8900 \\
\hline 30 & $\frac{7180069576834562839}{175111372800}$ & $4.10 \times 10^{7}$ & more than 82000000 \\
\hline 31 & $?$ & $>4 \times 10^{11}$ & more than $8 \times 10^{11}$ \\
\hline 32 & $?$ & $>5 \times 10^{15}$ & more than $1 \times 10^{16}$ \\
\hline 33 & $?$ & $>4 \times 10^{20}$ & more than $8 \times 10^{20}[8]$ \\
\hline$n$ & $m_{n}^{\mathrm{II}}(\emptyset)$ & (as decimal) & number of lattices \\
\hline 0 & 1 & 1 & (empty lattice) \\
\hline 8 & 0 & 0 & 0 \\
\hline 16 & 0 & 0 & 0 \\
\hline 24 & $\frac{1}{8315553613086720000}$ & $1.20 \times 10^{-19}$ & $1 \quad$ (Leech lattice) \\
\hline 32 & $\frac{1310037331282023326658917}{238863431761920000}$ & $5.48 \times 10^{6}$ & more than 10000000 \\
\hline
\end{tabular}

We can construct 32-dimensional odd unimodular lattices with no roots from 32 -dimensional even unimodular lattices with no roots as follows. Let $\Lambda$ be a 32 dimensional even unimodular lattice with no roots, and let $b \in \Lambda / 2 \Lambda$ be a nonzero element with norm divisible by 4 . Then there is a unique 32-dimensional odd lattice $L$ containing $\Lambda_{b}:=\{v \in \Lambda \mid(v, b) \in 2 Z\}$ (see [4, Chapter 0.2]). ( $\Lambda$ and $L$ are neighbors, meaning their intersection has index two in each of them.) If $b$ is not represented by a vector of norm 0 or 4 , then $L$ has no roots. Of the $2^{32}$ elements in $\Lambda / 2 \Lambda, 2^{31}+2^{15}$ have norm congruent to $0 \bmod 4$, by Milgram's formula [27. Appendix 4] applied to the lattice $\sqrt{2} \Lambda$. (Milgram's formula says that if $M$ is an even lattice and $M^{\prime}$ its dual, then

$$
\sum_{u \in M^{\prime} / M} \exp (2 \pi i(u, u) / 2)=(\operatorname{det} M)^{1 / 2} \exp (2 \pi i \sigma / 8),
$$

where $\sigma$ is the signature of $M$, which is equal to its dimension when $M$ is positive definite.) Note that $(b, b) \bmod 4$ depends only on $b \bmod 2 \Lambda$. Vectors $v$ and $w$ of norm 4 in $\Lambda$ are equivalent $\bmod 2 \Lambda$ if and only if $v= \pm w$, so $146880 / 2$ 
elements of $\Lambda / 2 \Lambda$ are represented by vectors of norm 4 . Thus there are $2^{31}+$ $2^{15}-146880 / 2-1=2147442975$ elements of $\Lambda / 2 \Lambda$ represented by vectors of norm divisible by 4 but not represented by vectors of norm 0 or 4 . Since each odd 32-dimensional unimodular lattice has two even neighbors, this implies that $m_{32}^{\mathrm{I}}(\emptyset) \geq(2147442975 / 2) \cdot m_{32}^{\mathrm{II}}(\emptyset)=5.89 \times 10^{15}$. (This is almost certainly an underestimate, since an even neighbor of an odd lattice with no roots can have roots.)

As an immediate consequence of the values we computed for $m_{n}(\emptyset)$ in Table 2 we have:

Corollary 13 ([9, 4, 2]). There exist odd unimodular $n$-dimensional lattices without roots for $n=23,24$, and $26, \ldots, 32$, but not for $n=1, \ldots, 22$ or $n=25$.

Unimodular lattices with no roots are known to exist in all dimensions $n \geq 26$ (see [8], [28]). In dimensions $n \leq 28$ they have already been completely enumerated, and so the masses $m_{n}(\emptyset)$ may also be computed by summing the reciprocals of the automorphism group orders of these lattices. (Happily, this agrees with our mass formula in each case.) For the even lattices, there is the empty lattice in dimension 0 , and the Leech lattice $\Lambda_{24}$ in dimension 24. The Leech lattice $\Lambda_{24}$ was discovered by Leech in 1965 25] and was shown to be the unique even unimodular 24-dimensional lattice without roots by Niemeier [29] and by Conway 9, Chapter 12 ] around 1969. For the odd lattices, there is the shorter Leech lattice in dimension 23, the odd Leech lattice $O_{24}$ in dimension 24 [30], the lattice $S_{26}$ in dimension 26, 3 lattices in dimension 27, and 38 lattices in dimension 28. The lattice $S_{26}$ was constructed by Conway in the 1970's and was shown to be the unique unimodular 26-dimensional lattice without roots by Borcherds in 1984 4. Borcherds also found one of the 27-dimensional lattices. The full enumerations in dimensions 27 and 28 are due to Bacher and Venkov [2].

Remark 14. In the cases of the Leech lattice and $S_{26}$, with our mass formula the uniqueness follows immediately from the constructions, simply by verifying that $\left|\operatorname{Aut}\left(\Lambda_{24}\right)\right|^{-1}=m_{24}^{\mathrm{II}}(\emptyset)$ and $\left|\operatorname{Aut}\left(S_{26}\right)\right|^{-1}=m_{26}^{\mathrm{I}}(\emptyset)$.

Examples of unimodular lattices with no roots have been constructed for dimensions 29 to 32 (including the 15 exceptional even unimodular 32-dimensional lattices classified in [24]), and a nonconstructive analytic argument shows that they exist in all dimensions $n \geq 33$ (see the Conway-Thompson Theorem [27, p. 46] for $n \geq 37$, and [7] for $33 \leq n \leq 36$.) In fact for $n \geq 33$ this argument gives a lower bound for the mass of lattices without roots that is close to the total mass of the genus, so there are a great many lattices without roots (see Remark 16). The idea is that the coefficients $a_{1}$ and $a_{2}$ of the average theta series

$$
\frac{1}{m} \sum_{\Lambda \in \Omega} \frac{\Theta_{\Lambda}(q)}{|\operatorname{Aut}(\Lambda)|}=1+a_{1} q^{1}+a_{2} q^{2}+a_{3} q^{3}+\cdots
$$

give the average number of vectors of norm 1 and norm 2 , taken over all the lattices in the genus $\Omega$. If $a_{1}+a_{2}$ is less than 2 , then there must be some $\Lambda \in \Omega$ with no vectors of norm 1 or 2 - this is the case when $\Omega$ is the genus of $n$-dimensional unimodular lattices for $n \geq 33$. For $n=33, a_{1}+a_{2}$ is approximately 1.42 , and this implies that $m_{33}^{\mathrm{I}}(\emptyset) \geq 4.04 \times 10^{20}$, so there are more than $8 \times 10^{20} 33$-dimensional unimodular lattices without roots [8]. For $n \leq 32$, the average number of roots is greater than 2, so this argument does not apply. Notice that the coefficient $a_{i}$ is 
the same as the average number of representations $a(N)$ we defined in Section 2 in the special case where $N$ is the $1 \times 1$ matrix $(i)$.

Remark 15. In dimensions $n \leq 33$, odd unimodular lattices with no roots have minimal norm 3, except in dimension 32 , in which they can have minimal norm 3 or 4 . In dimensions 24 and 32 , even unimodular lattices with no roots have minimal norm 4. See 8].

Remark 16. Let $m_{n}^{\prime}$ be the mass of odd $n$-dimensional unimodular lattices with only trivial automorphisms. There are no such lattices for $n \leq 28$ [2], but Bacher has found one for $n=29$ [1]. Bannai [3] showed that $m_{n}^{\prime} / m_{n} \rightarrow 1$ as $n \rightarrow \infty$. For $n>1$ any lattice with roots has nontrivial automorphisms, so $m_{n}^{\prime} / m_{n} \leq m_{n}(\emptyset) / m_{n}$. Below we list $m_{n}(\emptyset) / m_{n}$ for $26 \leq n \leq 30$, and lower bounds on $m_{n}(\emptyset) / m_{n}$ for $31 \leq n \leq 33$.

\begin{tabular}{|c|c|c|c|c|c|}
\hline$n$ & $m_{n}(\emptyset) / m_{n}$ & ref. & $n$ & $m_{n}(\emptyset) / m_{n}$ & ref. \\
\hline 26 & 0.000116 & {$[4$} & 30 & 0.0908 & \\
\hline 27 & 0.000856 & 2 & 31 & $>0.135$ & \\
\hline 28 & 0.00658 & 2 & 32 & $>0.136$ & \\
\hline 29 & 0.0300 & & 33 & $>0.287$ & 8 \\
\hline
\end{tabular}

\section{LOWER BOUNDS ON CLASS NUMBERS}

Let $\Omega$ be the set of inequivalent lattices in a genus of dimension $n>0, m$ the mass of that genus, and $m(R)$ the mass of those lattices having root system $R$. Each lattice $\Lambda \in \Omega$ has at least two automorphisms, $1: x \mapsto x$ and $-1: x \mapsto-x$; from this we get the well-known lower bound $|\Omega| \geq\lceil 2 m\rceil$. For each root $r$ of $\Lambda$, the reflection

$$
x \mapsto x-2 \frac{(x, r)}{(r, r)} r
$$

is also in $\operatorname{Aut}(\Lambda)$. Define $w^{\prime}(R)$ to be the order of the subgroup of Aut $(\Lambda)$ generated by reflections and by -1 . Then $w^{\prime}(R)=w(R)$ if the map -1 is already in the Weyl group of $R$, and $w^{\prime}(R)=2 w(R)$ otherwise. (The Weyl group of $R$ contains -1 if and only if $\operatorname{rank}(R)=\operatorname{dim}(\Lambda)$ and each component of $R$ is $A_{1}, E_{7}, E_{8}$ or $D_{k}$ for even $k$.) Then there are at least $\left\lceil m(R) w^{\prime}(R)\right\rceil$ lattices with root system $R$, so we get an improved lower bound,

$$
|\Omega| \geq \sum_{R}\left\lceil m(R) w^{\prime}(R)\right\rceil
$$

We can do slightly better still, as follows: Write $m(R) w^{\prime}(R)=q+a / b$ with $q, a, b \in \mathrm{Z}, a<b$, and $\operatorname{gcd}(a, b)=1$, and define a modified ceiling function by $\langle q+a / b\rangle=q$ if $a=0, q+1$ if $a=1$, and $q+2$ if $a>1$. It can easily be shown that there are at least $\left\langle m(R) w^{\prime}(R)\right\rangle$ lattices with root system $R$, so that

$$
|\Omega| \geq \sum_{R}\left\langle m(R) w^{\prime}(R)\right\rangle .
$$

Evaluating this sum with the value of $m_{32}^{\mathrm{II}}(R)$ we computed for each $R$ gives $|\Omega| \geq 1162109024$.

Corollary 17. There are at least one billion even unimodular 32-dimensional lattices. 
TABLE 3. Comparison of the actual number $\alpha_{n}$ of unimodular lattices of dimension $n$, the lower bound $\beta_{n}$ computed from (11), the number $r_{n}$ of distinct root systems occuring (including those with components Z), and the Minkowski-Siegel mass constant $m_{n}$. Odd lattices are listed above, even lattices below. The last column is included only when $2 m_{n}$ provides a nontrivial lower bound for $\alpha_{n}$.

\begin{tabular}{|c|c|c|c|c|c|c|}
\hline $\operatorname{dim} n$ & actual $\alpha_{n}^{\mathrm{I}}$ & $\geq$ & bound $\beta_{n}^{\mathrm{I}}$ & $r_{n}^{\mathrm{I}}$ & $\operatorname{mass} m_{n}^{\mathrm{I}}$ & $\beta_{n}^{\mathrm{I}} / 2 m_{n}^{\mathrm{I}}$ \\
\hline 0 & 0 & $\geq$ & 0 & 0 & 0 & \\
\hline 1 & 1 & $\geq$ & 1 & 1 & 0.5 & \\
\hline 2 & 1 & $\geq$ & 1 & 1 & 0.125 & \\
\hline 3 & 1 & $\geq$ & 1 & 1 & $2.083 \times 10^{-2}$ & \\
\hline 4 & 1 & $\geq$ & 1 & 1 & $2.604 \times 10^{-3}$ & \\
\hline 5 & 1 & $\geq$ & 1 & 1 & $2.604 \times 10^{-4}$ & \\
\hline 6 & 1 & $\geq$ & 1 & 1 & $2.170 \times 10^{-5}$ & \\
\hline 7 & 1 & $\geq$ & 1 & 1 & $1.551 \times 10^{-6}$ & \\
\hline 8 & 1 & $\geq$ & 1 & 1 & $9.688 \times 10^{-8}$ & \\
\hline 9 & 2 & $\geq$ & 2 & 2 & $6.100 \times 10^{-9}$ & \\
\hline 10 & 2 & $\geq$ & 2 & 2 & $4.485 \times 10^{-10}$ & \\
\hline 11 & 2 & $\geq$ & 2 & 2 & $4.213 \times 10^{-11}$ & \\
\hline 12 & 3 & $\geq$ & 3 & 3 & $5.267 \times 10^{-12}$ & \\
\hline 13 & 3 & $\geq$ & 3 & 3 & $9.031 \times 10^{-13}$ & \\
\hline 14 & 4 & $\geq$ & 4 & 4 & $2.186 \times 10^{-13}$ & \\
\hline 15 & 5 & $\geq$ & 5 & 5 & $7.705 \times 10^{-14}$ & \\
\hline 16 & 6 & $\geq$ & 6 & 6 & $4.093 \times 10^{-14}$ & \\
\hline 17 & 9 & $\geq$ & 9 & 9 & $3.402 \times 10^{-14}$ & \\
\hline 18 & 13 & $\geq$ & 13 & 13 & $4.583 \times 10^{-14}$ & \\
\hline 19 & 16 & $\geq$ & 16 & 16 & $1.033 \times 10^{-13}$ & \\
\hline 20 & 28 & $\geq$ & 28 & 28 & $4.002 \times 10^{-13}$ & \\
\hline 21 & 40 & $\geq$ & 40 & 40 & $2.735 \times 10^{-12}$ & \\
\hline 22 & 68 & $\geq$ & 68 & 68 & $3.377 \times 10^{-11}$ & \\
\hline 23 & 117 & $\geq$ & 117 & 117 & $7.710 \times 10^{-10}$ & \\
\hline 24 & 273 & $\geq$ & 273 & 266 & $3.330 \times 10^{-8}$ & \\
\hline 25 & 665 & $\geq$ & 657 & 609 & $2.781 \times 10^{-6}$ & \\
\hline 26 & $?$ & $\geq$ & 2307 & 1695 & $4.586 \times 10^{-4}$ & \\
\hline 27 & $?$ & $\geq$ & 14179 & 4492 & $1.524 \times 10^{-1}$ & \\
\hline 28 & $?$ & $\geq$ & 327972 & 9213 & $1.040 \times 10^{2}$ & 1569.2 \\
\hline 29 & $?$ & $\geq$ & 37938009 & 20298 & $1.486 \times 10^{5}$ & 127.7 \\
\hline 30 & $?$ & $\geq$ & 20169641025 & 67848 & $4.520 \times 10^{8}$ & 22.3 \\
\hline 31 & $?$ & $\geq$ & $?$ & $?$ & $2.980 \times 10^{12}$ & \\
\hline 32 & $?$ & $\geq$ & $?$ & $?$ & $4.328 \times 10^{16}$ & \\
\hline $\operatorname{dim} n$ & actual $\alpha_{n}^{\mathrm{II}}$ & $\geq$ & bound $\beta_{n}^{\mathrm{II}}$ & $r_{n}^{\mathrm{II}}$ & mass $m_{n}^{\mathrm{II}}$ & $\beta_{n}^{\mathrm{II}} / 2 m_{n}^{\mathrm{II}}$ \\
\hline 0 & 1 & $\geq$ & 1 & 1 & 1 & \\
\hline 8 & 1 & $\geq$ & 1 & 1 & $1.435 \times 10^{-9}$ & \\
\hline 16 & 2 & $\geq$ & 2 & 2 & $2.489 \times 10^{-18}$ & \\
\hline 24 & 24 & $\geq$ & 24 & 24 & $7.937 \times 10^{-15}$ & \\
\hline 32 & $?$ & $\geq$ & 1162109024 & 13218 & $4.031 \times 10^{7}$ & 14.4 \\
\hline
\end{tabular}


For comparison, $\left\lceil 2 m_{32}^{\mathrm{II}}\right\rceil=80618466$, so our lower bound is 14.4 times larger than the lower bound obtained by doubling the Minkowski-Siegel mass constant.

We also computed $m_{n}(R)$ for each $n \leq 30$ and each root system $R$, and used (II) to find lower bounds on the number of odd unimodular lattices. (In the cases where a single $m_{n}(R)$ is expressed as a sum of masses corresponding to different root systems of 32-dimensional even unimodular lattices, such as $m_{28}(\emptyset)=$ $m_{32}^{\mathrm{II}}\left(D_{4}\right) 3 w\left(D_{4}\right)+m_{32}^{\mathrm{II}}\left(D_{5}\right) w\left(D_{5}\right)$, we bounded the number for each summand individually, which gives a better overall bound.)

Unimodular lattices in dimensions $n \leq 25$ have been completely enumerated: even unimodular lattices of dimension 8 by Mordell, of dimension 16 by Witt, and of dimension 24 by Niemeier 29] (see also Venkov 33); odd unimodular lattices of dimension $n \leq 16$ by Kneser [23], of dimension $n \leq 23$ by Conway and Sloane 9 . Chapter 16], and of dimension 24 and 25 by Borcherds [4].

Table 3 gives our lower bound $\beta_{n}$ on the number of unimodular lattices in dimension $n \leq 30$, and our computation of the number of distinct root systems $r_{n}$ that occur in these lattices (including root systems with components Z, which do not occur for even lattices). The table includes for comparison the actual number $\alpha_{n}$ of unimodular lattices in dimension $n \leq 25$, taken from [9, Table 2.2], and the Minkowski-Siegel mass constants $m_{n}$, taken from [9, Tables 16.3 and 16.5]. (The counts of odd unimodular lattices include those with vectors of norm 1 to facilitate comparison with $m_{n}$; since any integral lattice with a vector of norm 1 is of the form $\mathrm{Z}^{k} \oplus \Lambda$ where $\Lambda$ has minimal (nonzero) norm 2 , the counts of lattices with no vectors of norm 1 can be recovered from $\beta_{n}$; similarly, the number of distinct root systems with no components $\mathrm{Z}$ can be recovered from $r_{n}$, with the caution that 8 of the 24 root systems of even unimodular 24-dimensional lattices also occur as root systems of odd unimodular 24-dimensional lattices.) Our lower bounds agree exactly with the actual numbers for $n \leq 24$. This is to be expected for $n \leq 23$ since in those dimensions an odd or even $n$-dimensional unimodular lattice is uniquely determined by its root system $R$, and $\left\langle m(R) w^{\prime}(R)\right\rangle$ is exactly equal to the number of lattices with root system $R$ when there are 0 or 1 such lattices. This is also true for 24-dimensional even unimodular lattices. Our lower bound in dimension 25 is within two percent of the actual number, and our lower bounds in dimensions 26 to 30 (for which the actual numbers are not known) are the best we are aware of.

\section{Computing the numbers $a(R)$}

For a half-integral $n \times n$ matrix $B$, define

$$
c_{n, k}(B)=(-1)^{n k / 2} 2^{n(k-(n-1) / 2)}(\operatorname{det} B)^{(2 k-n-1) / 2} b(B, k) \prod_{i=2 k-n+1}^{2 k} \frac{\pi^{i / 2}}{\Gamma(i / 2)},
$$

where

$$
b(B, k)=\sum_{R \in S_{n}(\mathrm{Q}) / S_{n}(\mathrm{Z})} \exp (2 \pi i \operatorname{tr}(B R)) \mu(R)^{-k}
$$

is the Siegel series (with $\mu(R)$ equal to the product of denominators of elementary divisors of $R$ ). Put $\epsilon_{n, k}=1 / 2$ if $n=k-1$ or $n=k>1$, and 1 otherwise. 
Theorem 18 (Siegel). Let $N$ be a lattice with $\operatorname{dim}(N)=n \leq 8 k$, and let

$$
a(N)=\frac{1}{m_{8 k}^{I I}} \sum_{\Lambda \in \Omega} \frac{r(\Lambda, N)}{|\operatorname{Aut}(\Lambda)|}
$$

with the sum taken over even unimodular lattices of dimension $8 k$. Then $a(N)=$ $\epsilon_{n, 8 k} c_{n, 4 k}(B)$, where $B$ is $1 / 2$ times the Gram matrix of $N$.

Proof. See [21, Theorem 6.8.1], and note that the product of local densities

$$
\prod_{p} \alpha_{p}\left(M_{p}, N_{p}\right)
$$

is equal to $b(M, k)$ when $N$ is an even unimodular lattice of dimension $2 k$ [20].

Remark 19. The Siegel Eisenstein series of degree $n$ and weight $k$ is defined to be

$$
E_{n, k}(Z)=\sum_{\{C, D\}}|C Z+D|^{-k}
$$

where $\{C, D\}$ runs over all representatives of the equivalence classes of coprime pairs of $n \times n$ matrices. For $k>n, c_{n, k}(C)$ is the coefficient in the Fourier expansion

$$
E_{n, k}(Z)=\sum_{C} c_{n, k}(C) \exp (2 \pi i \operatorname{tr}(C Z)),
$$

where $C$ runs over all positive semi-definite half-integral $n \times n$ matrices.

The first explicit formula for $b(B, k)$ for arbitrary $n$ is due to Katsurada [15], and was published in 1999. Prior to this, an explicit formula for the coefficients of $E_{n, k}(Z)$ was known only for $n \leq 3$ : The case $n=1$ is well-known (see for example [32, Chapter VII]), Maaß 26] gives an explicit formula for $n=2$ (see also Kaufhold [16]), and Katsurada [14 gives an explicit formula for $n=3$ (extending partial results by Kitaoka [19]).

Let $B$ be a nondegenerate symmetric half-integral $n \times n$ matrix over $\mathrm{Z}_{p}$. It follows from [22] that

$$
b(B, s)=\left(\zeta(s) \prod_{i=1}^{\lfloor r / 2\rfloor} \zeta(2 s-2 i)\right)^{-1} \prod_{p \mid D(B)} F_{p}\left(B ; p^{-s}\right) \times \begin{cases}L\left(s-r / 2 ; \chi_{B}\right) & \text { if } r \text { is even, } \\ 1 & \text { if } r \text { is odd }\end{cases}
$$

for certain polynomials $F_{p}(B ; X)$. Here $\zeta$ is the Riemann zeta function, and $L\left(s, \chi_{B}\right)$ is a Dirichlet $L$-series whose values may be computed using the method in 7 .

The explicit formula for $F_{p}(B ; X)$ in [15, Theorem 4.3] is not itself well-suited for calculation, since the outer index of summation takes $2^{n}$ values, but we can use Katsurada's recursion relations [15, Theorems 4.1 and 4.2] as part of a practical algorithm for computing $F_{p}(B ; X)$. We will state these recursion relations below (without proof); first we will need to introduce some of the notation from [15].

For $a=p^{r} c$ with $r \in \mathrm{Z}$ and $c \in \mathrm{Z}_{p}^{*}$, define $\chi_{p}(a)=\left(\frac{c}{p}\right)$ for $r$ even and 0 for $r$ odd (where $(\bar{p})$ is the Legendre symbol $\bmod p$ ), and define

$$
\chi_{2}(a)= \begin{cases}+1 & \text { if } r \equiv 0 \bmod 2, c \equiv 1 \bmod 8 \\ -1 & \text { if } r \equiv 0 \bmod 2, c \equiv 5 \bmod 8 \\ 0 & \text { otherwise. }\end{cases}
$$

Define $\operatorname{ord}_{p}(a)$ to be the exact power of $p$ dividing $a$, and define $i_{p}(B)$ to be the least integer $t$ for which $p^{t} B^{-1}$ is half-integral. Let $(,)_{p}$ denote the Hilbert symbol 
over $\mathrm{Q}_{p}$ (see [32]), and let $h_{p}$ denote the Hasse invariant (see [21]). For odd $n$, define

$$
\eta_{p}(B)=h_{p}(B)\left(\operatorname{det} B,(-1)^{(n-1) / 2} \operatorname{det} B\right)_{p}(-1,-1)_{p}^{\left(n^{2}-1\right) / 8}
$$

For even $n$, define

$$
\xi_{p}(B)=\chi_{p}\left((-1)^{n / 2} \operatorname{det} B\right)
$$

and $\xi_{p}^{\prime}(B)=1+\xi_{p}(B)-\xi_{p}(B)^{2}$. By convention, $\xi_{p}(B)=\xi_{p}^{\prime}(B)=1$ for $B$ the empty matrix. Define $D(B)=2^{2\lfloor n / 2\rfloor} \operatorname{det} B, d_{p}(B)=\operatorname{ord}_{p}(D(B))$, and

$$
\delta_{p}(B)= \begin{cases}2\left\lfloor\left(d_{p}(B)+1-\delta_{2 p}\right) / 2\right\rfloor & \text { if } n \text { is even, } \\ d_{p}(B) & \text { if } n \text { is odd, }\end{cases}
$$

where $\delta_{2 p}$ is the Kronecker delta.

We shall suppress most of the subscripts $p$ in what follows. Let $p$ be any prime and suppose $B$ and $B_{2}$ are nondegenerate half-integral matrices of rank $n$ and $n-1$ respectively over $\mathrm{Z}_{p}$. Put $\delta=\delta(B)$ and $\tilde{\delta}=\delta\left(B_{2}\right)$. If $n$ is even, put $\xi=\xi(B)$, $\xi^{\prime}=\xi^{\prime}(B)$, and $\tilde{\eta}=\eta\left(B_{2}\right)$; if $n$ is odd, put $\tilde{\xi}=\xi\left(B_{2}\right), \tilde{\xi}^{\prime}=\xi^{\prime}\left(B_{2}\right)$, and $\eta=\eta(B)$. (By convention $\tilde{\eta}=\tilde{\eta}^{\prime}=1$ if $B_{2}=\emptyset$, and $\tilde{\delta}=0$ if $n=1$.) Then define rational functions $C\left(B, B_{2} ; X\right)^{(1)}$ and $C\left(B, B_{2} ; X\right)^{(0)}$ in $X$ by

$$
C\left(B, B_{2} ; X\right)^{(1)}= \begin{cases}\frac{1-p^{n / 2} \xi X}{1-p^{n+1} X^{2}} & \text { if } n \text { is even, } \\ \frac{1}{1-p^{(n+1) / 2} \xi X} & \text { if } n \text { is odd }\end{cases}
$$

and

$$
C\left(B, B_{2} ; X\right)^{(0)}= \begin{cases}\frac{\left.(-1)^{\xi+1} \xi^{\prime} \tilde{\eta}\left(1-p^{n / 2+1} X \xi\right)\left(p^{n / 2} X\right)^{\delta-\tilde{\delta}+\xi^{2}} p^{\delta / 2}\right)}{1-p^{n+1} X^{2}} & \text { if } n \text { is even, } \\ \frac{(-1)^{\tilde{\xi}} \tilde{\xi}^{\prime} \eta\left(p^{(n-1) / 2} X\right)^{\delta-\tilde{\delta}+2-\tilde{\xi}^{2}} p^{(2 \delta-\tilde{\delta}+2) / 2}}{1-p^{(n+1) / 2} \tilde{\xi} X} & \text { if } n \text { is odd. }\end{cases}
$$

Theorem 20 (Katsurada [15]). Let $B_{1}=\left(b_{1}\right)$ and $B_{2}$ be nondegenerate half-integral matrices of degree 1 and $n-1$, respectively, over $\mathrm{Z}_{p}$, and put $B=B_{1} \perp B_{2}$. Assume that ord $\left(b_{1}\right) \geq i\left(B_{2}\right)-1+2 \delta_{2 p}$. Then we have

$$
F_{p}(B ; X)=C\left(B, B_{2} ; X\right)^{(1)} F\left(B_{2} ; p X\right)+C\left(B, B_{2} ; X\right)^{(0)} F_{p}\left(B_{2} ; X\right) .
$$

Let $B_{2}$ be a nondegenerate half-integral matrix of degree $n-2$ over $\mathrm{Z}_{2}$, let $H=\left(\begin{array}{ll}0 & 1 / 2 \\ 1 / 2 & 0\end{array}\right)$ and let $Y=\left(\begin{array}{ll}1 & 1 / 2 \\ 1 / 2 & 1\end{array}\right)$. Let $B_{1}=2^{m} K$ with $K=H$ or $Y$, or $B_{1}=$ $2^{m} u_{1} \perp 2^{m} u_{2}$ with $u_{1}, u_{2} \in \mathrm{Z}_{2}^{*}$, and put $B=B_{1} \perp B_{2}, \delta=\delta(B), \tilde{\delta}=\delta\left(2^{m} \perp B_{2}\right)$, $\hat{\delta}=\delta\left(B_{2}\right)$, and

$$
\sigma= \begin{cases}(2 \tilde{\delta}-\delta-\hat{\delta}+2) / 2 & \text { if } n \text { is even, } B_{1}=2^{m} u_{1} \perp 2^{m} u_{2} \text { and } d(B) \text { is odd } \\ 2 & \text { or if } n \text { is even, } B_{1}=2^{m} K, \text { and } \xi\left(B_{2}\right)=0, \\ 0 & \text { if } n \text { is odd, } B_{1}=2^{m} K, \text { and } d\left(2^{m} \perp B_{2}\right) \text { is even, } \\ \text { otherwise. }\end{cases}
$$

If $n$ is even, put $\xi=\xi(B), \xi^{\prime}=\xi^{\prime}(B), \hat{\xi}=\xi\left(B_{2}\right), \hat{\xi}^{\prime}=\xi^{\prime}\left(B_{2}\right)$, and

$$
\tilde{\eta}= \begin{cases}\eta\left(2^{m} u_{2} \perp B_{2}\right) & \text { if } B_{1}=2^{m} u_{1} \perp 2^{m} u_{2} \\ (-1)^{\left((n-1)^{2}-1\right) / 8} h\left(B_{2}\right)\left(2^{m},(-1)^{(n-1) / 2} \operatorname{det} B_{2}\right)_{2} & \text { and } B_{1}=2^{m} K \\ & \text { and } \xi\left(B_{2}\right) \neq 0 \\ 1 & \text { otherwise }\end{cases}
$$


If $n$ is odd, put $\eta=\eta(B), \hat{\eta}=\eta\left(B_{2}\right), \tilde{\xi}^{\prime}=1$, and $\tilde{\xi}=1$ if $B_{1}=2^{m} K$ and $d\left(2^{m} \perp B_{2}\right)$ is even, and 0 otherwise. Define four rational functions in $X$ by

$$
\begin{gathered}
C\left(B, B_{2} ; X\right)^{(11)}= \begin{cases}\frac{1-2^{n / 2} \xi X}{1-2^{n+1} X^{2}} & \text { if } n \text { is even, } \\
\frac{1-2^{(n+1) / 2} \tilde{\xi} X}{\text { if } n \text { is odd, }}\end{cases} \\
C\left(B, B_{2} ; X\right)^{(10)}= \begin{cases}\frac{\left.(-1)^{\xi+1} \xi^{\prime} \tilde{\eta}\left(1-2^{n / 2+1} X \xi\right)\left(2^{n / 2} X\right)^{\delta-\tilde{\delta}+\xi^{2}+\sigma} 2^{\delta / 2}\right)}{1-2^{n+1} X^{2}} & \text { if } n \text { is even, } \\
\frac{(-1)^{\tilde{\xi}} \eta\left(2^{(n-1) / 2} X\right)^{\delta-\tilde{\delta}+2-\tilde{\xi}^{2}+\sigma} 2^{(2 \delta-\tilde{\delta}+2+\sigma) / 2}}{1-2^{(n+1) / 2} \tilde{\xi} X} & \text { if } n \text { is odd, }\end{cases} \\
C\left(B, B_{2} ; X\right)^{(21)}= \begin{cases}\frac{1}{1-2^{n / 2} \hat{\xi} X} & \text { if } n \text { is even, } \\
\frac{1-2^{(n-1) / 2} \tilde{\xi} X}{1-2^{n} X^{2}} & \text { if } n \text { is odd, }\end{cases} \\
C\left(B, B_{2} ; X\right)^{(20)}= \begin{cases}\frac{(-1)^{\hat{\xi}} \hat{\xi} \tilde{\eta}\left(2^{(n-2) / 2} X\right)^{\tilde{\delta}-\hat{\delta}+2-\hat{\xi}^{2}-\sigma} 2^{(2 \tilde{\delta}-\hat{\delta}+2-2 \sigma) / 2}}{1-2^{n / 2} \hat{\xi} X} & \text { if } n \text { is even, } \\
\frac{\left.(-1)^{\tilde{\xi}+1} \hat{\eta}\left(1-2^{(n+1) / 2} X \tilde{\xi}\right)\left(2^{(n-1) / 2} X\right)^{\tilde{\delta}-\hat{\delta}+\tilde{\xi}^{2}-\sigma} 2^{(\tilde{\delta}-\sigma) / 2}\right)}{1-2^{n} X^{2}} & \text { if } n \text { is odd. }\end{cases}
\end{gathered}
$$

Theorem 21 (Katsurada [15). Let $B_{1}=2^{m} u_{1} \perp 2^{m} u_{2}$ with $u_{1}, u_{2} \in \mathrm{Z}_{2}^{*}$ or $B=$ $2^{m} K$ with $K=H$ or $Y$. Let $B_{2}$ be a half-integral matrix of degree $n-2$ over $\mathrm{Z}_{2}$ which is also in $G L_{n-2}\left(\mathrm{Q}_{2}\right)$, and put $B=B_{1} \perp B_{2}$. Assume that $m \geq i\left(B_{2}\right)+1$. Then we have

$$
\begin{aligned}
F_{2}(B ; X) & =C\left(B, B_{2} ; X\right)^{(11)} C\left(B, B_{2} ; 2 X\right)^{(21)} F_{2}\left(B_{2} ; 4 X\right) \\
& +C\left(B, B_{2} ; X\right)^{(11)} C\left(B, B_{2} ; 2 X\right)^{(20)} F_{2}\left(B_{2} ; 2 X\right) \\
& +C\left(B, B_{2} ; X\right)^{(10)} C\left(B, B_{2} ; X\right)^{(21)} F_{2}\left(B_{2} ; 2 X\right) \\
& +C\left(B, B_{2} ; X\right)^{(10)} C\left(B, B_{2} ; X\right)^{(20)} F_{2}\left(B_{2} ; X\right) .
\end{aligned}
$$

For $p \neq 2$, any nondegenerate symmetric half-integral $n \times n$ matrix $B$ can be diagonalized over the $p$-adic integers $\mathrm{Z}_{p}, B \cong p^{e_{1}} u_{1} \perp \ldots \perp p^{e_{n}} u_{n}$ with $e_{1} \geq \cdots \geq$ $e_{n} \geq 0$ and with $u_{i} \in\{1, \epsilon\}$ for all $i$, where $\epsilon$ is any quadratic nonresidue (see [34]). Then $F_{p}(B ; X)$ can be computed by repeated applications of Theorem 20 above.

Note that in the course of recursively computing $F_{p}(B ; X)$ for $X=p^{-k}$ in this manner, one occasionally encounters zeros of the denominators of the functions $C^{(1)}$ and $C^{(0)}$. (This is only a problem when $X$ is a negative power of $p$.) Rather than attempting to simplify the expressions symbolically, we instead computed $F_{p}(B ; 1), F_{p}(B ; p), \ldots, F_{p}\left(B ; p^{n}\right)$, and then used Lagrangian interpolation to compute $F_{p}\left(B ; p^{-k}\right)$. The reason for choosing $X=1, p, \ldots, p^{n}$ rather than $n+1$ other numbers is that for these numbers the recursive subproblems overlap: we need only evaluate $F_{p}\left(p^{e_{i}} u_{i} \perp \ldots \perp p^{e_{n}} u_{n} ; X\right)$ for $X=1, p, \ldots, p^{2 n-i}$, starting with $i=n$, and working down to $i=1$, which gives $F_{p}(B ; 1), \ldots, F_{p}\left(B ; p^{n}\right)$.

For $p=2$, any nondegenerate symmetric half-integral matrix $B$ is equivalent over $\mathrm{Z}_{2}$ to a matrix of the form $2^{e_{1}}\left(U_{1} \perp V_{1}\right) \perp \ldots \perp 2^{e_{m}}\left(U_{m} \perp V_{m}\right)$ with $e_{1}>\cdots>$ $e_{m} \geq 0, U_{i}=\emptyset$ or $u_{1}$ or $u_{1} \perp u_{2}$ for $u_{1}, u_{2} \in\{ \pm 1, \pm 3\}$, and $V_{i}=\emptyset$ or $H \perp \cdots \perp H$ or $H \perp \cdots \perp H \perp Y$ (see [34]). Then $F_{2}(B ; X)$ can be computed by repeated applications of Theorems 20 and 21 above. (We again used an interpolation scheme, similar to the one described for $p \neq 2$.) 


\section{Computing the numbers $r\left(R_{i}, R_{j}\right)$}

The method described in this section is essentially the one used for some of the computations in [6] (although the algorithm itself is not described in that paper). In this section we write $r\left(R, R^{\prime}\right)$ as $\operatorname{emb}\left(R^{\prime}, R\right)$, since a representation of $R^{\prime}$ by $R$ is the same as a linear map from $R^{\prime}$ into $R$ that preserves inner products. For any irreducible root systems $S$ and $T$ it is routine to compute the number of embeddings emb $(S, T)$ of $S$ into $T$, and to determine the root system of the orthogonal complement of $S$ in $T$ for each of these embeddings. There are at most two orbits of embeddings of $S$ into $T$, so we write $\operatorname{emb}(S, T)=\operatorname{emb}_{1}(S, T)+\operatorname{emb}_{2}(S, T)$, where there are $\operatorname{emb}_{1}(S, T)$ embeddings of $S$ into $T$ for which the orthogonal complement is $\operatorname{comp}_{1}(S, T)$ and there are $\operatorname{emb}_{2}(S, T)$ embeddings of $S$ into $T$ for which the orthogonal complement is $\operatorname{comp}_{2}(S, T)$.

Example 22. We demonstrate how to compute the two orbits of embeddings of $A_{3}$ into $D_{n}$. Recall from [9] that $A_{n}=\left\{\left(x_{1}, \ldots, x_{n+1}\right) \in \mathrm{Z}^{n+1}: x_{1}+\cdots+x_{n+1}=0\right\}$ and $D_{n}=\left\{\left(x_{1}, \ldots, x_{n}\right) \in \mathrm{Z}^{n}: x_{1}+\cdots+x_{n} \in 2 \mathrm{Z}\right\} . A_{3}$ is generated by three roots $v_{1}=(1,-1,0,0), v_{2}=(0,1,-1,0)$, and $v_{3}=(0,0,1,-1)$ with $\left(v_{1}, v_{1}\right)=\left(v_{2}, v_{2}\right)=$ $\left(v_{3}, v_{3}\right)=2,\left(v_{1}, v_{2}\right)=\left(v_{2}, v_{3}\right)=-1$ and $\left(v_{1}, v_{3}\right)=0$. We can map $v_{1}$ to any of the $4 C(n, 2)$ roots $r_{1}$ in $D_{n}$ (all permutations of $( \pm 1, \pm 1,0, \ldots, 0)$ ), where $C(n, k)$ denotes the binomial coefficient.

We can then map $v_{2}$ to any of the $4(n-2)$ roots $r_{2}$ of $D_{n}$ that have inner product -1 with $r_{1}$. Let us say $r_{1}$ is supported in coordinates $i$ and $j$ and $r_{2}$ is supported in coordinates $j$ and $k$. (Clearly $k \neq i$.) Then there are two cases:

(a) We can map $v_{3}$ to the root $r_{3}$ supported in coordinates $i$ and $j$ that has inner product 0 with $r_{1}$ and inner product -1 with $r_{2}$. In this case the roots of $D_{n}$ orthogonal to $r_{1}, r_{2}$, and $r_{3}$ form the system $D_{n-3}$.

(b) We can map $v_{3}$ to $2(n-3)$ roots $r_{3}$ supported in coordinates $k$ and $l(l \neq i, j)$ that have inner product 0 with $r_{1}$ and inner product -1 with $r_{2}$. In this case the roots of $D_{n}$ orthogonal to $r_{1}, r_{2}$, and $r_{3}$ form the system $D_{n-4}$.

Hence there are $4 C(n, 2) \cdot 4(n-2) \cdot 1=C(n, 3) \cdot\left|\operatorname{Aut}\left(A_{3}\right)\right|$ ways to embed $A_{3}$ into $D_{n}$ with complement $D_{n-3}$, and there are $4 C(n, 2) \cdot 4(n-2) \cdot 2(n-3)=$ $2^{3} C(n, 4) \cdot\left|\operatorname{Aut}\left(A_{3}\right)\right|$ ways to embed $A_{3}$ into $D_{n}$ with complement $D_{n-4}$.

The computations for other irreducible root systems are similar. All nonzero values of emb ${ }_{1}, \mathrm{emb}_{2}, \mathrm{comp}_{1}$, and $\mathrm{comp}_{2}$ are given in Table 4

If $R$ and $R^{\prime}$ are root lattices, then we can write $R=S_{1} \oplus \cdots \oplus S_{k}$ and $R^{\prime}=$ $T_{1} \oplus \cdots \oplus T_{m}$, where each $S_{i}$ and $T_{i}$ is an irreducible root lattice, and we can compute $r\left(R^{\prime}, R\right)$ recursively via the formula

$$
\begin{aligned}
r\left(R^{\prime}, R\right) & =\operatorname{emb}\left(R, R^{\prime}\right) \\
& =\operatorname{emb}\left(\bigoplus_{i \leq k} S_{i}, \bigoplus_{i \leq m} T_{i}\right) \\
& =\sum_{j=1}^{m} \operatorname{emb}_{1}\left(S_{k}, T_{j}\right) \operatorname{emb}\left(\bigoplus_{i \leq k-1} S_{i}, \operatorname{comp}_{1}\left(S_{k}, T_{j}\right) \oplus \bigoplus_{i \leq m, i \neq j} T_{i}\right) \\
& +\sum_{j=1}^{m} \operatorname{emb}_{2}\left(S_{k}, T_{j}\right) \operatorname{emb}\left(\bigoplus_{i \leq k-1} S_{i}, \operatorname{comp}_{2}\left(S_{k}, T_{j}\right) \oplus \bigoplus_{i \leq m, i \neq j} T_{i}\right) .
\end{aligned}
$$


TABLE 4. Embeddings of irreducible root systems into one another. (In the fourth and last columns, $A_{0}, D_{0}, D_{1}, D_{2}$ and $D_{3}$ should be interpreted as $\emptyset, \emptyset, \emptyset, A_{1} A_{1}$ and $A_{3}$, respectively.)

\begin{tabular}{|c|c|c|c|c|c|}
\hline$S$ & $T$ & $\frac{\operatorname{emb}_{1}(S, T)}{|\operatorname{Aut}(S)|}$ & $\operatorname{comp}_{1}(S, T)$ & $\frac{\frac{\operatorname{emb}_{2}(S, T)}{|\operatorname{Aut}(S)|}}{\mid \sin ^{\prime}(S)}$ & $\operatorname{comp}_{2}(S, T)$ \\
\hline$\emptyset$ & $T$ & 1 & $T$ & - & - \\
\hline$A_{i}$ & $A_{j}(j \geq i)$ & $C(j+1, i+1)$ & $A_{j-i-1}$ & - & - \\
\hline$A_{1}$ & $D_{j}$ & $C(j, 2) \cdot 2$ & $A_{1} D_{j-2}$ & - & - \\
\hline$A_{3}$ & $D_{j}$ & $C(j, 4) \cdot 2^{3}$ & $D_{j-4}$ & $C(j, 3)$ & $D_{j-3}$ \\
\hline$A_{i}(i \neq 1,3)$ & $D_{j}(j>i)$ & $C(j, i+1) \cdot 2^{i}$ & $D_{j-i-1}$ & - & - \\
\hline$D_{i}$ & $D_{j}(j \geq i)$ & $C(j, i)$ & $D_{j-i}$ & - & - \\
\hline$A_{1}$ & $E_{6}$ & $2^{2} 3^{2}$ & $A_{5}$ & - & - \\
\hline$A_{2}$ & $E_{6}$ & $2^{3} 3 \cdot 5$ & $A_{2} A_{2}$ & - & - \\
\hline$A_{3}$ & $E_{6}$ & $2 \cdot 3^{3} 5$ & $A_{1} A_{1}$ & - & - \\
\hline$A_{4}$ & $E_{6}$ & $2^{3} 3^{3}$ & $A_{1}$ & - & - \\
\hline$A_{5}$ & $E_{6}$ & $2^{2} 3^{2}$ & $A_{1}$ & - & - \\
\hline$D_{4}$ & $E_{6}$ & $3^{2} 5$ & $\emptyset$ & - & - \\
\hline$D_{5}$ & $E_{6}$ & $3^{3}$ & $\emptyset$ & - & - \\
\hline$E_{6}$ & $E_{6}$ & 1 & $\emptyset$ & - & - \\
\hline$A_{1}$ & $E_{7}$ & $3^{2} 7$ & $D_{6}$ & - & - \\
\hline$A_{2}$ & $E_{7}$ & $2^{4} 3 \cdot 7$ & $A_{5}$ & - & - \\
\hline$A_{3}$ & $E_{7}$ & $2^{2} 3^{2} 5 \cdot 7$ & $A_{3} A_{1}$ & - & - \\
\hline$A_{4}$ & $E_{7}$ & $2^{5} 3^{2} 7$ & $A_{2}$ & - & - \\
\hline$A_{5}$ & $E_{7}$ & $2^{4} 3 \cdot 7$ & $A_{2}$ & $2^{4} 3^{2} 7$ & $A_{1}$ \\
\hline$A_{6}$ & $E_{7}$ & $2^{5} 3^{2}$ & $\emptyset$ & - & - \\
\hline$A_{7}$ & $E_{7}$ & $2^{2} 3^{2}$ & $\emptyset$ & - & - \\
\hline$D_{4}$ & $E_{7}$ & $3^{2} 5 \cdot 7$ & $A_{1} A_{1} A_{1}$ & - & - \\
\hline$D_{5}$ & $E_{7}$ & $2 \cdot 3^{3} 7$ & $A_{1}$ & - & - \\
\hline$D_{6}$ & $E_{7}$ & $3^{2} 7$ & $A_{1}$ & - & - \\
\hline$E_{6}$ & $E_{7}$ & $2^{2} 7$ & $\emptyset$ & - & - \\
\hline$E_{7}$ & $E_{7}$ & 1 & $\emptyset$ & - & - \\
\hline$A_{1}$ & $E_{8}$ & $2^{3} 3 \cdot 5$ & $E_{7}$ & - & - \\
\hline$A_{2}$ & $E_{8}$ & $2^{5} 5 \cdot 7$ & $E_{6}$ & - & - \\
\hline$A_{3}$ & $E_{8}$ & $2^{3} 3^{3} 5 \cdot 7$ & $D_{5}$ & - & - \\
\hline$A_{4}$ & $E_{8}$ & $2^{7} 3^{3} 7$ & $A_{4}$ & - & - \\
\hline$A_{5}$ & $E_{8}$ & $2^{7} 3^{2} 5 \cdot 7$ & $A_{2} A_{1}$ & - & - \\
\hline$A_{6}$ & $E_{8}$ & $2^{8} 3^{3} 5$ & $A_{1}$ & - & - \\
\hline$A_{7}$ & $E_{8}$ & $2^{5} 3^{3} 5$ & $A_{1}$ & $2^{6} 3^{3} 5$ & $\emptyset$ \\
\hline$A_{8}$ & $E_{8}$ & $2^{6} 3 \cdot 5$ & $\emptyset$ & - & - \\
\hline$D_{4}$ & $E_{8}$ & $2 \cdot 3^{2} 5^{2} 7$ & $D_{4}$ & - & - \\
\hline$D_{5}$ & $E_{8}$ & $2^{3} 3^{3} 5 \cdot 7$ & $A_{3}$ & - & - \\
\hline$D_{6}$ & $E_{8}$ & $2^{2} 3^{3} 5 \cdot 7$ & $A_{1} A_{1}$ & - & - \\
\hline$D_{7}$ & $E_{8}$ & $2^{3} 3^{3} 5$ & $\emptyset$ & - & - \\
\hline$D_{8}$ & $E_{8}$ & $3^{3} 5$ & $\emptyset$ & - & - \\
\hline$E_{6}$ & $E_{8}$ & $2^{5} 5 \cdot 7$ & $A_{2}$ & - & - \\
\hline$E_{7}$ & $E_{8}$ & $2^{3} 3 \cdot 5$ & $A_{1}$ & - & - \\
\hline$E_{8}$ & $E_{8}$ & 1 & $\emptyset$ & - & - \\
\hline
\end{tabular}


A direct implementation of this algorithm does a lot of redundant computation on certain inputs, some of which we can circumvent with dynamic programming or memoization (see [10, Chapter 16]). Our implementation also does several things to reduce the amount of computation when there are direct summands in $R$ or $R^{\prime}$ with multiplicity greater than one. But computing $r\left(R^{\prime}, R\right)$ is an NP-hard problem, since it is an NP-complete problem to determine if $r\left(R^{\prime}, R\right)>0$, as we show below.

Proposition 23. The problem of determining whether a root system $R$ embeds into a root system $R^{\prime}$ is NP-complete.

Proof. We shall reduce 3-PARTition (see [13]) to this problem. Let $S=\left\{s_{1}, \ldots\right.$, $\left.s_{3 k}\right\}$ be an instance of 3-PARTITION, with the $s_{i}$ positive integers summing to $k t$. Then $S$ can be partitioned into $k$ sets each consisting of 3 elements with sum $t$ if and only if the root system $D_{4 s_{1}} \oplus \cdots \oplus D_{4 s_{3 k}}$ embeds into the root system $\bigoplus_{i=1}^{k} D_{4 t}$.

Remark 24. Since 3-PARTITION is strongly NP-complete, the problem of whether one root system embeds into another remains NP-complete if the Gram matrices of root lattices are used as input (rather that the list of components $A_{i}, D_{i}$, and $E_{i}$ as above).

Since we need to compute $r\left(R, R^{\prime}\right)$ for all pairs of root systems rather than just one pair, the amortized computational cost would be reduced considerably by using dynamic programming. But the dynamic programming table becomes unmanageably large in dimension 32 , so we instead use a hash table, which is purged periodically, for memoization.

\section{Eliminating Root Systems a priori}

There are 405844 root systems of rank $n \leq 32$ with no vectors of norm 1 , corresponding to all direct sums of $A_{i}, D_{i}(i \geq 4), E_{6}, E_{7}$ and $E_{8}$, where the order of the summands does not matter and the sum of the subscripts is at most 32 . Since computing the number of embeddings $r\left(R_{i}, R_{j}\right)$ can be time-consuming, we used the following congruences, due to Borcherds [4, to eliminate some root systems from consideration:

Let $\operatorname{roots}(R)$ denote the number of roots of $R$. If $R$ is the root system of a 32-dimensional even unimodular lattice, then:

if $R$ contains $E_{8}$, then $\operatorname{roots}(R) \equiv 0(\bmod 24)$,

if $R$ contains $E_{7}$, then $\operatorname{roots}(R) \equiv 0(\bmod 12)$,

if $R$ contains $E_{6}$, then $\operatorname{roots}(R) \equiv 0(\bmod 6)$,

if $R$ contains $D_{6}$, then $\operatorname{roots}(R) \equiv 0(\bmod 4)$,

if $R$ contains $D_{7}$, then $\operatorname{roots}(R) \equiv 0(\bmod 8)$,

if $R$ contains $D_{8}$, then $\operatorname{roots}(R) \equiv 0(\bmod 8)$,

if $R$ contains $D_{n}, n>8$, then $\operatorname{roots}(R) \equiv 0(\bmod 16)$.

Also note that if $a(R)=0$, then $m(R)=0$. If $\operatorname{rank}(R)=32$ and $\operatorname{det}(R)$ is not a perfect square, then $a(R)$ must be 0 , so we eliminated those root systems as well. This left 135443 root systems. We ordered them so that $\operatorname{dim}\left(R_{i}\right) \leq \operatorname{dim}\left(R_{j}\right)$ if $i<j$, and so that $\operatorname{det}\left(R_{i}\right) \geq \operatorname{det}\left(R_{j}\right)$ if $i<j$ and $\operatorname{dim}\left(R_{i}\right)=\operatorname{dim}\left(R_{j}\right)$. 
As the matrix $U$ with $U_{i, j}=r\left(R_{i}, R_{j}\right)$ would still contain around 10 billion elements, we did not explicitly construct and invert it. Rather, we computed each element in the matrix when it was required for solving $\frac{1}{m} U v=w$ by back-substitution, with

$$
m\left(R_{i}\right)=\frac{1}{r\left(R_{i}, R_{i}\right)}\left\{m \cdot a\left(R_{i}\right)-\sum_{j>i} r\left(R_{j}, R_{i}\right) m\left(R_{j}\right)\right\} .
$$

If $m\left(R_{j}\right)$ has already been computed to be 0 , then the values $r\left(R_{j}, R_{i}\right)$ need not be computed, since they make no contribution to this sum.

\section{ACKNOWLEDGMENTS}

The author would like to thank Richard Borcherds for many helpful suggestions, Richard Fateman for advice on Lisp, and Xerox PARC for the use of its computers.

\section{REFERENCES}

1. R. Bacher, Unimodular lattices without nontrivial automorphisms, Internat. Math. Res. Notes 2 (1994) 91-95. MR 95b:11067

2. R. Bacher and B. B. Venkov, Réseaux entiers unimodulaires sans racines en dimension 27 et 28, Réseaux euclidiens, designs sphériques et formes modulaires, 212-267, Monogr. Enseign. Math., 37, Enseignement Math., Geneva, 2001.

3. E. Bannai, Positive definitive unimodular lattices with trivial automorphism group, Mem. Amer. Math. Soc. 429 (1990) 1-70. MR 90j:11030

4. R. E. Borcherds, The Leech lattice and other lattices, Ph.D. Dissertation, University of Cambridge, 1984. Available at arXiv:math.NT/9911195 Much of this material also appears in [5].

5. R. E. Borcherds, Classification of positive definite lattices, Duke Math. J. 105 (2000), no. 3, 525-567. Available at arXiv:math. NT/9912236 MR 2001k:11057

6. R. E. Borcherds, E. Freitag and R. Weissauer, A Siegel cusp form of degree 12 and weight 12, J. Reine Angew. Math. 494 (1998) 141-153. MR 99d:11047 Available at arXiv:math.AG/9805132

7. J. H. Conway and N. J. A. Sloane, Low-dimensional lattices IV: The mass formula, Proc. R. Soc. Lond. A 419 (1988), 259-286. MR 90a:11074

8. J. H. Conway and N. J. A. Sloane, A note on optimal unimodular lattices, J. Number Theory 72 (1998), 357-362. MR 99k:11104

9. J. H. Conway and N. J. A. Sloane, Sphere Packings, Lattices and Groups, Springer-Verlag, NY, 3rd edition, 1998. MR 2000b:11077

10. T. H. Cormen, C. E. Leiserson and R. L. Rivest, Introduction to Algorithms, MIT Press, Cambridge, MA, 1990. MR 91i:68001

11. N. D. Elkies, Lattices and codes with long shadows, Math. Res. Lett. 2 (1995) 643-651. MR 96h:11065

12. N. D. Elkies and B. H. Gross, The exceptional cone and the Leech lattice, Internat. Math. Res. Notices 14 (1996), 665-698. MR 97g:11070

13. M. R. Garey and D. S. Johnson, Computers and Intractability: A Guide to the Theory of NP-Completeness, Freeman, SF, 1979. MR 80g:68056

14. H. Katsurada, An explicit formula for the Fourier coefficients of Siegel-Eisenstein series of degree 3, Nagoya Math J. 146 (1997), 199-223. MR 98g:11051

15. H. Katsurada, An explicit formula for Siegel series, Amer. J. Math. 121 (1999), 415-452. MR 2000a:11068

16. G. Kaufhold, Dirichletsche Reihe mit Funktionalgleichung in der Theorie der Modulfunktion 2: Grades, Math. Ann. 137 (1959), 454-476. MR 22:12223

17. M. Kervaire, Unimodular lattices with a complete root system, L'Enseign. Math. 40 (1994), 59-140. MR 95g:11063

18. O. D. King, Table of masses of even unimodular 32-dimensional lattices with any given root system. Available at arXiv:math.NT/0012231 
19. Y. Kitaoka, A note on local densities of quadratic forms, Nagoya Math J. 92 (1983), 145-152. MR 85e:11029

20. Y. Kitaoka, Local densities of quadratic forms and Fourier coefficients of Eisenstein series, Nagoya Math J. 103 (1986), 149-160. MR 87m:11041

21. Y. Kitaoka, Arithmetic of Quadratic Forms, Cambridge Tracts in Math., vol. 106, Cambridge Univ. Press, Cambridge, 1993. MR 95c:11044

22. Y. Kitaoka, Dirichlet series in the theory of Siegel modular forms, Nagoya Math J. 95 (1984), 73-84. MR 86b:11038

23. M. Kneser, Klassenzahlen definiter quadratischer Formen, Arch. Math. 8 (1957), 241-250. MR 19:838c

24. H. Koch and B. B. Venkov, Über ganzzahlige unimodulare euklidische Gitter, J. Reine Angew. Math. 398 (1989), 144-168. MR 90g:11082

25. J. Leech, Notes on sphere packings, Canadian J. Math. 19 (1967), 251-267. MR 35:878

26. H. Maaß, Die Fourierkoeffizienten der Eisensteinreihen zweiten Grades, Mat. Fys. Medd. Dan. Vid. Selsk. 34 (1973), 1-13. MR 58:22129

27. J. Milnor and D. Husemoller, Symmetric Bilinear Forms, Springer-Verlag, Berlin 1973.

28. G. Nebe and N. J. A. Sloane, A Catalogue of Lattices, published electronically at http://www.research.att.com/ njas/lattices/

29. H.-V. Niemeier, Definite quadratische Formen der Dimension 24 und Diskriminante 1, J. Number Theory 5 (1973), 142-178. MR 47:4931

30. R. E. O'Connor and G. Pall, The construction of integral quadratic forms of determinant 1, Duke Math. J. 11 (1944), 319-331. MR 5:254e

31. M. Peters, On even unimodular 32-dimensional lattices, Preprint SFB 478, Mathematischen Instituts der Westfälischen Wilhelms-Universität Münster, January 2001.

32. J.-P. Serre, A Course in Arithmetic, Springer-Verlag, NY 1973. MR 49:8956

33. B. B. Venkov, The classification of integral even unimodular 24-dimensional quadratic forms. Trudy Matematicheskogo Instituta imeni V. A. Steklova 148 (1978), 65-76. Also Chapter 18 of [9]; also Proc. Steklov Inst. Math. 1980, no. 4 (148), 63-74. MR 81d:11024

34. G. L. Watson, Integral Quadratic Forms, Cambridge Univ. Press, Cambridge, 1960. MR 22:9475

Department of Mathematics, University of California, Berkeley, California 94720

Current address: Department of Biological Chemistry and Molecular Pharmacology, Harvard

Medical School, 250 Longwood Avenue, SGMB-322, Boston, Massachusetts 02115

E-mail address: ok@csua.berkeley.edu 